\title{
OPEN The gut microbiota metabolite urolithin A inhibits NF-KB activation in LPS stimulated BMDMs
}

\author{
Khalid N. M. Abdelazeem ${ }^{1,2,3}$, M. Zaher Kalo ${ }^{3}$, Sandra Beer-Hammer ${ }^{3 凶}$ \& Florian Lang $^{1}$
}

Inflammation is a natural defense process of the innate immune system, associated with the release of proinflammatory cytokines such as interleukin-1 $\beta$, interleukin- 6 , interleukin-12 and TNF $\alpha$; and enzymes including iNOS through the activation and nuclear translocation of NF-kB p65 due to the phosphorylation of $\mathrm{I} K \mathrm{~B} \alpha$. Regulation of intracellular $\mathrm{Ca}^{2+}$ is considered a promising strategy for the prevention of reactive oxygen species (ROS) production and accumulation of DNA double strand breaks (DSBs) that occurs in inflammatory-associated-diseases. Among the metabolites of ellagitannins that are produced in the gut microbiome, urolithin A (UA) has received an increasing attention as a novel candidate with anti-inflammatory and anti-oxidant effects. Here, we investigated the effect of UA on the suppression of pro-inflammatory molecules and NF-KB activation by targeting TLR4 signalling pathway. We also identified the influence of UA on $\mathrm{Ca}^{2+}$ entry, ROS production and DSBs availability in murine bone-marrow-derived macrophages challenged with lipopolysaccharides (LPS). We found that UA inhibits IKB $\alpha$ phosphorylation and supresses MAPK and PI3K activation. In addition, UA was able to reduce calcium entry, ROS production and DSBs availability. In conclusion, we suggest that urolithin $A$ is a promising therapeutic agent for treating inflammatory diseases through suppression of NF-KB and preserving DNA through maintaining intracellular calcium and ROS homeostasis.

Antibiotic exposure in early life can lead to long-term alterations in the diversity, composition, and metagenomics content of the gut microbiota that may contribute to later onset of inflammatory bowel disease (IBD) $)^{1,2}$. IBD is a chronic inflammation of the gastrointestinal tract (GIT) which includes Crohn's disease (CD) and ulcerative colitis (UC), and estimated to affect more than $0.4 \%$ of Europeans and North Americans ${ }^{3}$. It is well recognized that patients with IBD are at high risk for developing colorectal cancer $(\mathrm{CRC})^{4,5}$. The nuclear factor- $\kappa \mathrm{B}(\mathrm{NF}-\kappa \mathrm{B})$ signaling pathway plays a prominent role in the development and maintenance of most chronic diseases ${ }^{6}$. NF-kB is a key regulator and important player in linking inflammation to cancer development through its ability to upregulate various inflammatory molecules such as IL- $1 \beta$, IL- 6 and TNF $\alpha$. It is a matchmaker between inflammation, IBD, cancer and diabetes ${ }^{7,8}$.

Intestinal inflammation is affected by the powerful anti-inflammatory effects of polyphenolic compounds 9 . Gut microbiota is able to convert dietary components such as polyphenols to a spectrum of metabolites ${ }^{10,11}$. Importantly, the health benefits rendered by consumption of several natural plant products (e.g. pomegranates, walnuts and berries) have been associated with high levels of polyphenolic compounds, specifically ellagitannins and ellagic acid ${ }^{12,13}$. Acid hydrolysis of ellagitannins releases free ellagic acid (EA $)^{14}$ which are present in pomegranates, raspberries, strawberries, and walnuts ${ }^{15}$.

Ellagitannins-rich food has beneficial effects on IBD and other inflammatory diseases as arthritis and cancer ${ }^{16}$. The bioavailability of ellagitannins and ellagic acid is very low. They need further digestion by gut microbiota to produce bioactive molecules including urolithin compounds that can be easily absorbed ${ }^{14,17,18}$. In addition, only one in each three people has the microbiota that can perform this metabolism with maximum efficiency ${ }^{19}$. Short chain fatty acids (propionic acid, acetic acid and butyric acid) are one of the most popular metabolites of

\footnotetext{
${ }^{1}$ Department of Internal Medicine III, Eberhard Karls University of Tübingen, Tübingen, Germany. ${ }^{2}$ Radiation Biology Research Department, National Centre for Radiation Research and Technology, Atomic Energy Authority, Cairo, Egypt. ${ }^{3}$ Department of Pharmacology, Experimental Therapy and Toxicology, Institute of Experimental and Clinical Pharmacology and Pharmacogenomic, University of Tübingen, Wilhelmstrasse 56, 72074 Tübingen, Germany. ${ }^{\varpi}$ email: sandra.beer-hammer@uni-tuebingen.de
} 
commensal microbes in the intestine which can contribute positively to the development of adaptive immunity $\mathrm{T}$ regulatory (Treg) cells ${ }^{20}$. Butyrate induces Treg cell differentiation in the gut mucosa, which reduces intestinal inflammation via secretion of anti-inflammatory cytokines such as IL-10 $0^{21,22}$. Acetate has been recognised for its ability to reduce the autoimmune $\mathrm{CD}^{+} \mathrm{T}$ cells and thus inhibit inflammation in type 1 diabetes ${ }^{23}$.

Macrophages are one of the primary components of innate immune system, which plays a prominent role during the immune response to harmful stimuli such as bacteria and viruses ${ }^{24}$. Macrophages are widely spread throughout the tissues and produce various bioactive molecules which determine the final outcome of inflammation. Thus, therapeutic approaches targeting macrophages and their products can provide new opportunities for controlling inflammatory disease ${ }^{25}$. Lipopolysaccharide (LPS) is the main component within the cell wall of Gram negative bacteria. It has been conventionally used to mimic the real infection triggering the release of inflammatory cytokines such as IL-1 $\beta$, IL- 6 or TNFa through activation of TLR4 signalling ${ }^{26}$.

The innate immune system provides the macrophages with pattern recognition system (PRRs) on their surface through which macrophages can sense pathogens and damaged cells through damage associated molecular patterns (DAMPs) ${ }^{27}$. The most important type of PRRs are toll-like receptors (TLRs). Studies have proposed that TLR2, TLR4, TLR5, TLR7, and TLR9 are the main PRRs, which stimulate inflammatory responses following the exposure of innate immune cells to pathogens or damaged cells ${ }^{28-30}$. These TLRs, through the stimulation of myeloid differentiation primary response 88 (MyD88), induce the upregulation of MAPK, NF- $\kappa B$, and other transcription factors, leading to the secretion of pro-inflammatory cytokines, including IL-1, IL-6, IL-8, TNF, IL-33, and IFN- $\gamma^{30}$. TLR2 and TLR4 have gained enormous importance due to their extreme ability of identifying distinct molecular patterns from invading pathogens. These PRRs not only act as innate sensors but also shape and bridge innate and adaptive immune responses. Furthermore, they play a crucial role in regulating the balance between Th1 and Th2 type of response essential for the survivability of the host ${ }^{31}$. Therefore, in this study, we attempted to analyze the ability of gut microbiota metabolites of ellagitannins urolithin A (UA) in reduction of inflammation induced by LPS (mimic the real scenario of bacterial infection) by targeting TLR4 signalling pathway (MAPK, NF- $\kappa \mathrm{B}$, and PI3K), pro-inflammatory cytokines, intracellular calcium, cellular ROS production and DNA double strand breaks (DSBs) in murine BMDMs.

\section{Results}

Urolithin A diminished the inflammatory miRNA expression in LPS-stimulated murine BMDMs. MicroRNAs (miRNA) are emerging as central regulators of inflammation. The post-transcriptional regulation is an important control mechanism for the expression of genes involved in inflammation like cytokines and chemokines ${ }^{32,33}$. Therefore, the influence of UA $(25 \mu \mathrm{M}$ or $50 \mu \mathrm{M})$ on inflammatory miRNA (Fig. 1) such as miR-9 (a), miR-10 (b), miR-99b (c), miR-146a (d), and miR-155 (e) in LPS-stimulated BMDMs were investigated. BMDMs receiving UA $(25 \mu \mathrm{M}$ or $50 \mu \mathrm{M})$ alone did not record any significant changes in examined miRNA expression except a slight non-significant elevation in miR-99b expression, whereas LPSstimulation induced the expression of miR-10, miR-99b, miR-146a, and miR-155. The presented data demonstrate that treatment with UA $(25 \mu \mathrm{M}$ or $50 \mu \mathrm{M})$ induced a remarkable decrease in miR-10, miR-99b, miR-146a and miR-155 expression in LPS-stimulated BMDMs with values near to untreated control.

Urolithin A blunted cellular ROS production in LPS-stimulated murine BMDMs. Dysregulated ROS production can enhance tumor formation via the activation of various oncogenic signalling pathways, DNA mutation, immune escape, tumor microenvironment and metastasis ${ }^{34}$. Thus, the level of ROS production was evaluated using fluorescence microscopy and flow cytometry in LPS-stimulated murine BMDMs with or without UA $(25 \mu \mathrm{M}$ or $50 \mu \mathrm{M})$. Murine BMDMs were labeled with MitoSOX ${ }^{\mathrm{ix}}$ Red reagent, which can be detected in red fluorescence when oxidized by superoxide, and nuclei were stained with blue-fluorescent DAPI (Fig. 2). The obtained results show that neither UA nor DMSO alone induced any significant changes on superoxide production in murine BMDMs after $48 \mathrm{~h}$ (Supplementary Fig. 1). Data represented in Fig. 2a demonstrate that after $48 \mathrm{~h}$ of LPS stimulation, BMDMs exhibit red immunofluorescence, indicating a highly significant increase in superoxide production. UA $(25 \mu \mathrm{M}$ or $50 \mu \mathrm{M})$ was able to inhibit the superoxide production (Fig. $2 \mathrm{a}, \mathrm{b})$.

In parallel, we studied the effect of UA $(25 \mu \mathrm{M}$ or $50 \mu \mathrm{M})$ on ROS production using H2DCFDA dye after $48 \mathrm{~h}$ (Fig. 2c, d). The obtained results indicate that neither DMSO nor UA $(25 \mu \mathrm{M}$ or $50 \mu \mathrm{M})$ alone induced any significant changes in ROS production. UA was, however, able to abolish the remarkable elevation of ROS production induced by LPS after $48 \mathrm{~h}$ (Fig. 2c, d).

Urolithin A reduced intracellular $\mathrm{Ca}^{2+}$ concentration in LPS-stimulated murine BMDMs. As the interactions between ROS and calcium signaling can be considered as bidirectional, wherein ROS can regulate cellular calcium signaling and calcium signaling is essential for ROS production ${ }^{35}$. Thus, the influence of UA $(25 \mu \mathrm{M}$ or $50 \mu \mathrm{M})$ on calcium entry in LPS-stimulated BMDMs after $24 \mathrm{~h}$ and $48 \mathrm{~h}$ was evaluated. The calcium influx or altered intracellular calcium concentration can be measured using a cell permeant dye, Fluo-4, which becomes fluorescent upon binding to calcium.

Results represented in Fig. 3 reveal that UA $(25 \mu \mathrm{M}$ or $50 \mu \mathrm{M})$ was able to revoke the upregulation of intracellular calcium induced by LPS in BMDMs after $48 \mathrm{~h}$. While, after $24 \mathrm{~h}$ a non-significant increase was recorded (Fig. 3b). In addition, the obtained results show that neither DMSO nor UA $(25 \mu \mathrm{M}$ or $50 \mu \mathrm{M})$ alone induced any significant change during the time intervals.

Urolithin A induced prominent decrease in DNA double strand breaks (DSBs) in LPS-stimulated murine BMDMs. Oxidative stress induced by extracellular and intracellular production of ROS is a fundamental mechanism that contributes to DNA damage. Overproduction of ROS that exceeds defense mecha- 
a
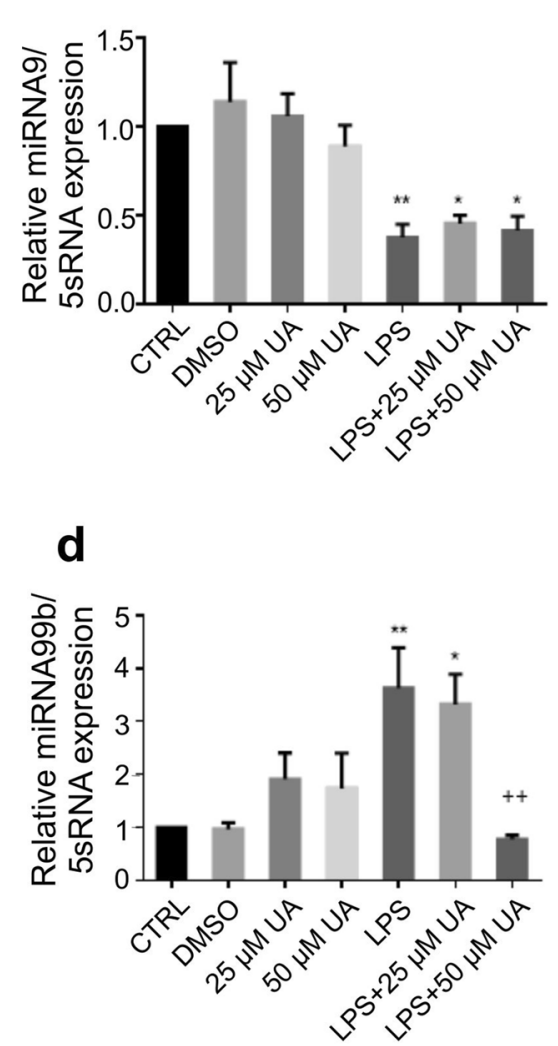

b

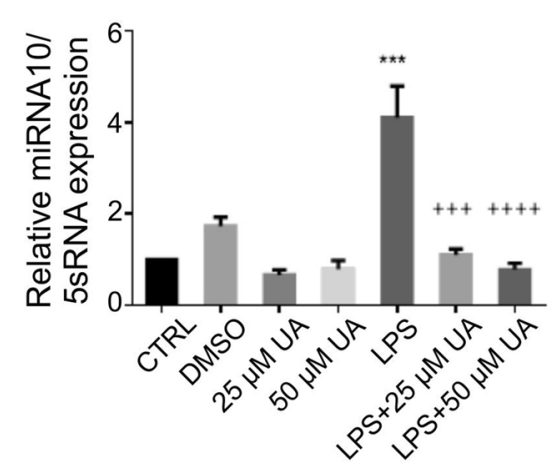

e

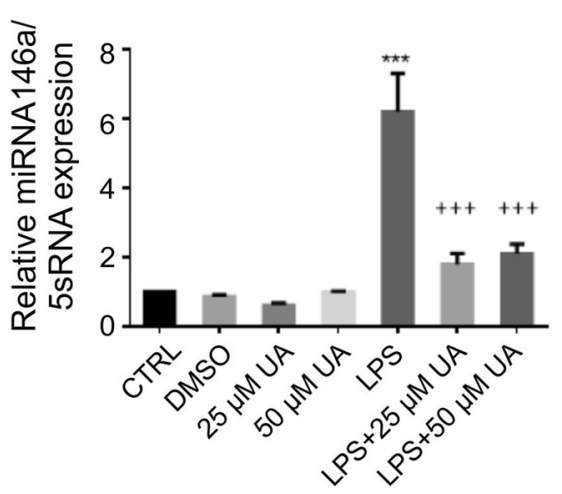

C

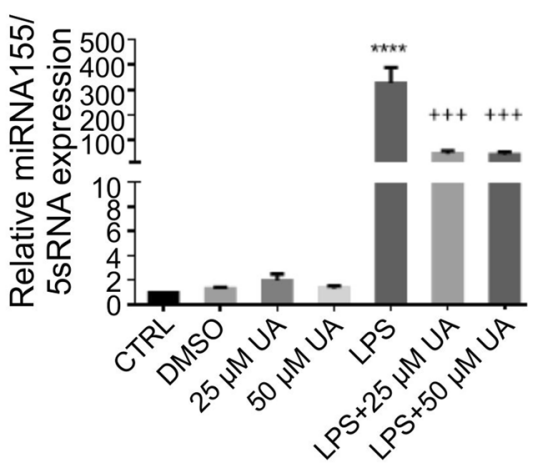

Figure 1. Urolithin A suppressed the inflammatory miRNA expression in LPS-stimulated murine BMDMs. Murine BMDMs were stimulated by $1 \mu \mathrm{g} / \mathrm{ml}$ of LPS in the presence or absence of UA $(25 \mu \mathrm{M}$ or $50 \mu \mathrm{M})$. The expression of miR-9 (a), miR-10 (b), miR-99b (c), miR.146a (d) and miR-155 (e) over 5S rRNA was evaluated in stimulated BMDMs after $72 \mathrm{~h}$. The unstimulated and untreated BMDMs were used as control. BMDMs treated with DMSO were used as negative control. Arithmetic means \pm SEM from seven independent experiments are depicted. One way ANOVA was used and ${ }^{\star}(p<0.05),{ }^{* *}(P 0.01)$, ${ }^{* * *}(p<0.001)$, and ${ }^{* * * *}(p<0.0001)$ indicate statistically significant differences compared to control. $++(P 0.01),+++(p<0.001)$, and $++++(p<0.0001)$ indicate statistically significant differences compared to LPS. Abbreviations: DMSO, dimethyl sulfoxide; UA, urolithin A; LPS, lipopolysaccharides; miR, micro RNA.

nisms can damage intracellular macromolecules, including nucleic acids, with the formation of DNA adducts and DNA strand breaks that may result in mutations ${ }^{36,37}$. As LPS induced upregulation of calcium entry and ROS production in BMDMs, we were interested in studying and evaluating the impact of LPS-stimulation on DSBs in the presence or absence of UA in BMDMs.

Murine BMDMs were fixed and immunostained for phosphorylated H2AX (Ser139) at $2 \mathrm{~h}$ (images are not shown) and $48 \mathrm{~h}$ (Fig. 3c) after LPS exposure. A higher significant number of $\gamma \mathrm{H} 2 \mathrm{AX}$ foci were only observed after $48 \mathrm{~h}$ (Fig. 3d) of LPS stimulation and UA was able to induce a prominent decrease in DSBs achieving values near to untreated control (Fig. 3d). In addition, neither UA $(25 \mu \mathrm{M}$ or $50 \mu \mathrm{M})$ nor DMSO alone induced any significant effect on DSBs in BMDMs (Supplementary Fig. 2).

Urolithin A downregulated the inflammatory cytokines production and mRNA expression in LPS-stimulated BMDMs. IL-1 $\beta$, IL-2, IL-6, IL-12 and TNFa (Fig. 4) are some of the pro-inflammatory cytokines that participate in the prolonging of chronic inflammation. These cellular messengers should be suppressed to avoid further inflammatory processes.

In addition, the anti-inflammatory IL-4, IL-10, IFN $\gamma$, TGF $\beta$ (Supplementary Fig. 3) as well as NOS2 (Fig. 4) were also evaluated either by ELISA and/or qRT-PCR in LPS-stimulated murine BMDMs. LPS is known to induce a typical M1 phenotype ${ }^{38}$. The presented data in Fig. 4 reveals that treatment with UA $(25 \mu \mathrm{M}$ or $50 \mu \mathrm{M})$ was able to induce significant depressions in IL-1 $\beta$ (a), IL-6 (b), IL-12 (c), TNF- $\alpha$ (d), and NOS2 (e) expression with values near to untreated control. On the other hand, the data presented in Supplementary Fig. 3 revealed that UA induced a remarkable decrease in IFN- $\gamma$ (a), TGF- $\beta$ (b), IL-10 (c), and IL-2 (d) expression, but a remarkable increase in IL-4 (e) expression was recorded in the presence of UA to LPS-stimulated BMDMs (Supplementary Fig. 3).

The notable increase in IL-10 expression was registered after $2 \mathrm{~h}$ and down regulated at later time points in the presence or absence of LPS and/or UA. Notably, LPS induced a significant elevation in the mRNA expression of IFN- $\gamma$ which did not translated into protein during the depicted time points as compared to their respective 


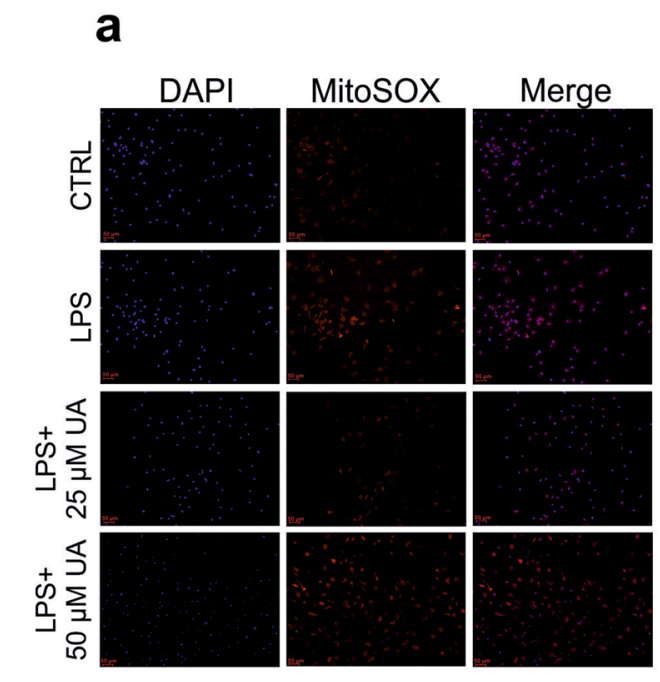

b

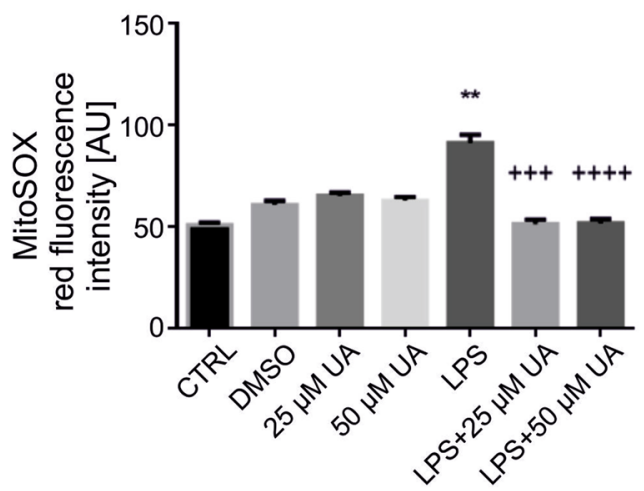

\section{C}

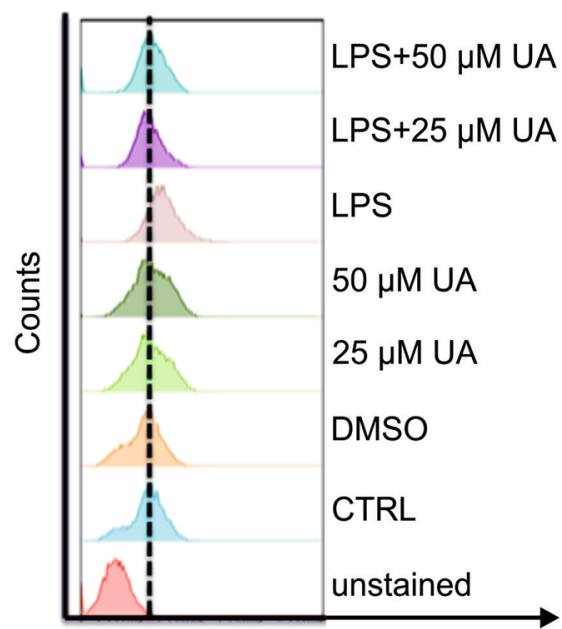

\section{d}

FL-1: FITC- ${ }_{2}$ DCFDA

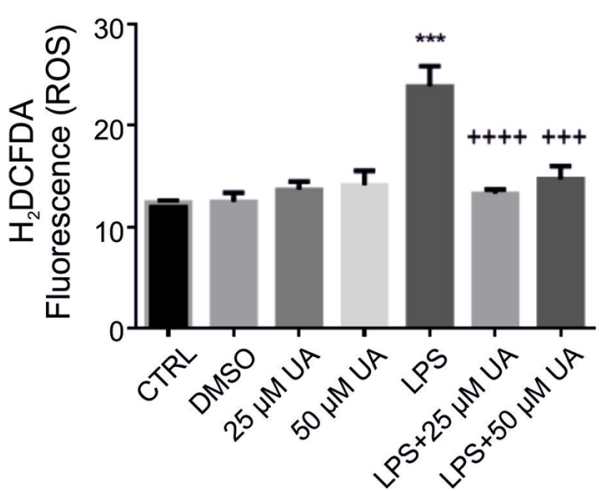

Figure 2. Urolithin A diminished cellular ROS production in LPS-stimulated murine BMDMs. (a) Murine BMDMs were stimulated by $1 \mu \mathrm{g} / \mathrm{ml}$ of LPS in presence or absence of UA $(25 \mu \mathrm{M}$ or $50 \mu \mathrm{M})$ for $48 \mathrm{~h}$. LPS induced remarkable elevation of superoxide production (MitoSOX) demonstrated by red immunofluorescence which canceled with UA. Nuclei were counterstained with DAPI (blue). Images were taken with fixed exposure times for DAPI $=6$ and MitoSOX $=750$. The magnifications are 20-fold and scale bar represents $50 \mu \mathrm{m}$. (b) The graph represents a significant difference in mitochondrial ROS production compared to untreated control. (c) Representative original FACS histograms showing the effect of UA on ROS production $\left(\mathrm{H}_{2} \mathrm{DCFDA}\right)$ in LPS-stimulated murine BMDMs after $48 \mathrm{~h}$. (d) The graph represents a significant difference in cellular ROS production compared to untreated control after $48 \mathrm{~h}$. The unstimulated and untreated murine BMDMs were used as control. Murine BMDMs treated with DMSO were used as negative control. Arithmetic means \pm SEM $(n=5-7)$. One way ANOVA was used and ${ }^{\star *}(P<0.001)$ and ${ }^{\star * *}(p<0.001)$ indicate statistically significant differences compared to control, whereas $+++(p<0.001)$, and $++++(p<0.0001)$ indicate statistically significant differences compared to LPS. Abbreviations: DMSO, dimethyl sulfoxide; UA, urolithin A; LPS, lipopolysaccharides.

control groups. The administration of UA $(25 \mu \mathrm{M}$ or $50 \mu \mathrm{M})$ alone induced a notable dose dependent increase in IL-1 $\beta$, IL-2, IL-4, IL-6, NOS2, TGF- $\beta$ and IFN- $\gamma$ mRNA expression.

Urolithin A reduced TLR4 expression in LPS-stimulated murine BMDMs. The presented data in Supplementary Fig. 4 reveals that the stimulation of BMDMs with $1 \mu \mathrm{g}$ of LPS induced significant elevation in TLR4 $(72 \mathrm{~h})$ expression. Administration of UA to LPS-stimulated BMDMs induced remarkable decreases 
a

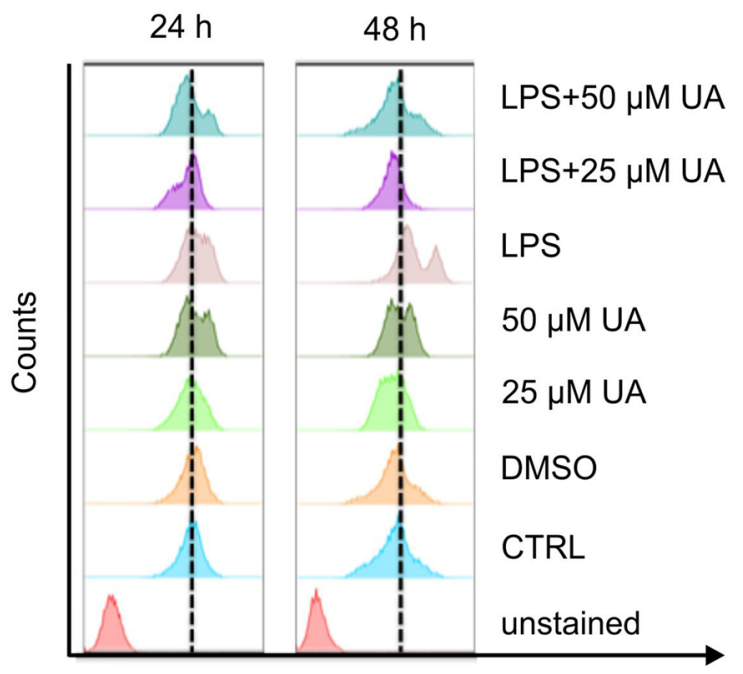

FL-1: FITC-FLUO4 b

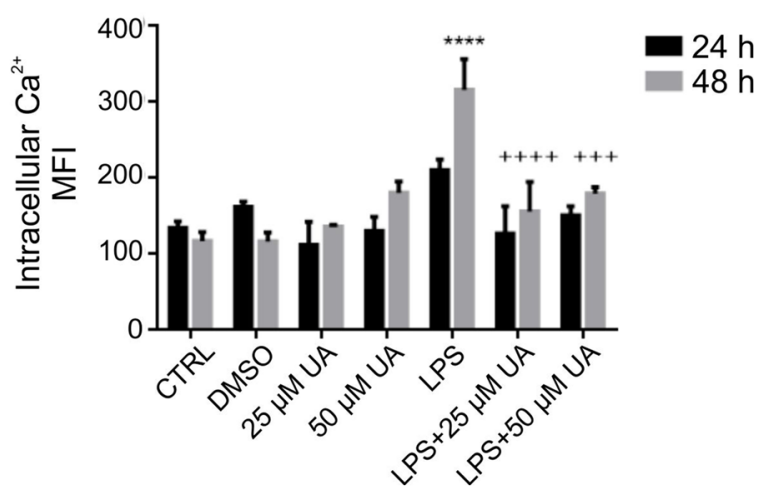

C

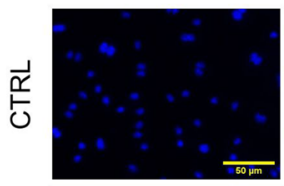

DAPI
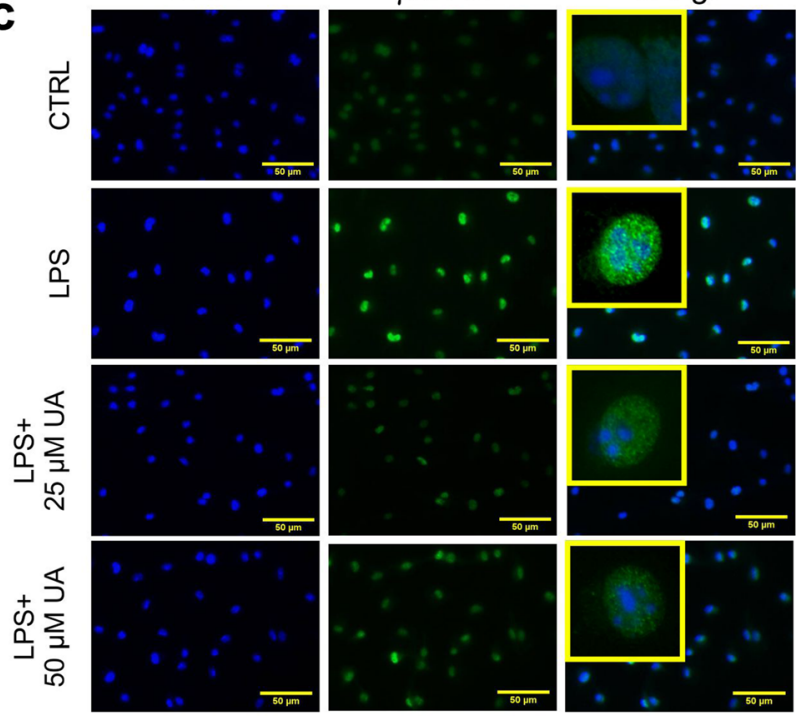

d

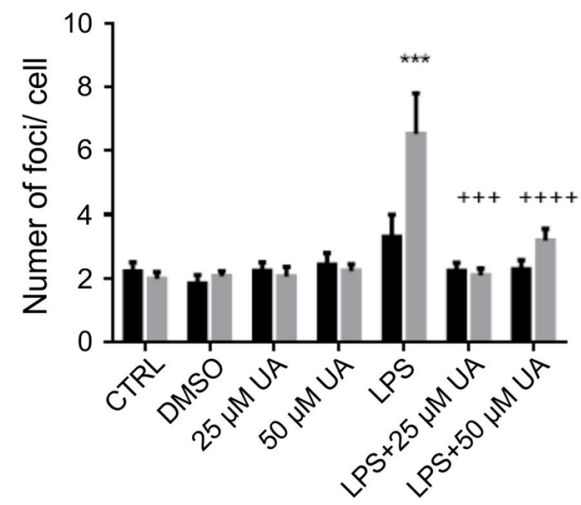

Figure 3. Urolithin A minimized the intracellular calcium concentration and ameliorated DSBs in LPSstimulated murine BMDMs. (a, b) Murine BMDMs were stimulated with $1 \mu \mathrm{g} / \mathrm{ml}$ LPS in the presence or absence of UA $(25 \mu \mathrm{M}$ or $50 \mu \mathrm{M})$ for $24 \mathrm{~h}$ or $48 \mathrm{~h}$. (a) Representative FACS histograms showing the effect of UA on stimulated BMDMs after $24 \mathrm{~h}$ and $48 \mathrm{~h}$. The reference line was set at the peak of the control. (b) Arithmetic means \pm SEM $(n=5-7)$ show a significant difference in intracellular $\mathrm{Ca}^{2+}$ concentration between control and treated groups after $24 \mathrm{~h}$ and $48 \mathrm{~h}$. The unstimulated BMDMs were used as untreated control. DMSO was used as negative control. The intracellular $\mathrm{Ca}^{2+}$ was measured with flow cytometry. (c) Murine BMDMs were stimulated by $1 \mu \mathrm{g} / \mathrm{ml}$ of LPS with or without UA $(25 \mu \mathrm{M}$ or $50 \mu \mathrm{M})$ for $2 \mathrm{~h}$ (images not shown) and $48 \mathrm{~h}$. After $48 \mathrm{~h}$, LPS induced DSBs indicated by a prominent green immunofluorescence for Ser139phosphorylated H2AX. Treatment with UA recorded a remarkable decrease in number of $\gamma \mathrm{H} 2 \mathrm{AX}$ foci. Nuclei were counterstained with DAPI (blue). The magnifications are 20 -fold and scale bar represents $50 \mu \mathrm{m}$. The unstimulated and untreated BMDMs were used as control. DMSO was used as negative control. (d) Graph indicates the number of $\gamma \mathrm{H} 2 \mathrm{AX}$ foci per cell after indicated time points. Representative images and arithmetic means \pm SEM from four independent experiments (600 cells were counted). Two way ANOVA was used and ${ }_{* * *}(p<0.001)$ indicate statistically significant differences compared to respective control, whereas $+++(p<0.001)$, and $++++(p<0.0001)$ indicate statistically significant differences compared to LPS. Abbreviations: DMSO, dimethyl sulfoxide; UA, urolithin A; LPS, lipopolysaccharides.

in TLR4 expression (Supplementary Fig. 4). Murine BMDMs receiving both concentrations of UA (25 $\mu$ M or $50 \mu \mathrm{M}$ ) alone had no significant effect on TLR4 expression.

Urolithin A revoked IKB $\alpha$ phosphorylation in LPS-stimulated murine BMDMs. NF- $\kappa B$ is the

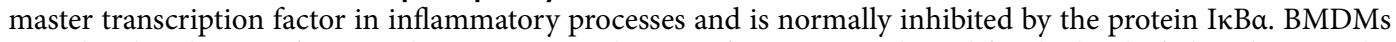
stimulated with $1 \mu \mathrm{g}$ of LPS in the presence or absence of UA were evaluated for the level of phospho-IкBa by immunoblot analysis. Data presented in Fig. 5 show that UA $(25 \mu \mathrm{M}$ or $50 \mu \mathrm{M})$ was able to impair the upregu-

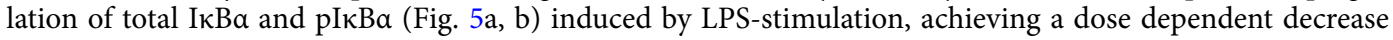
in $\mathrm{pI \kappa \textrm {B }} \alpha$ compared to those receiving LPS alone during the time intervals. A representative image of protein expression at $2 \mathrm{~h}$ and $72 \mathrm{~h}$ is shown in Supplementary Fig. 5. 

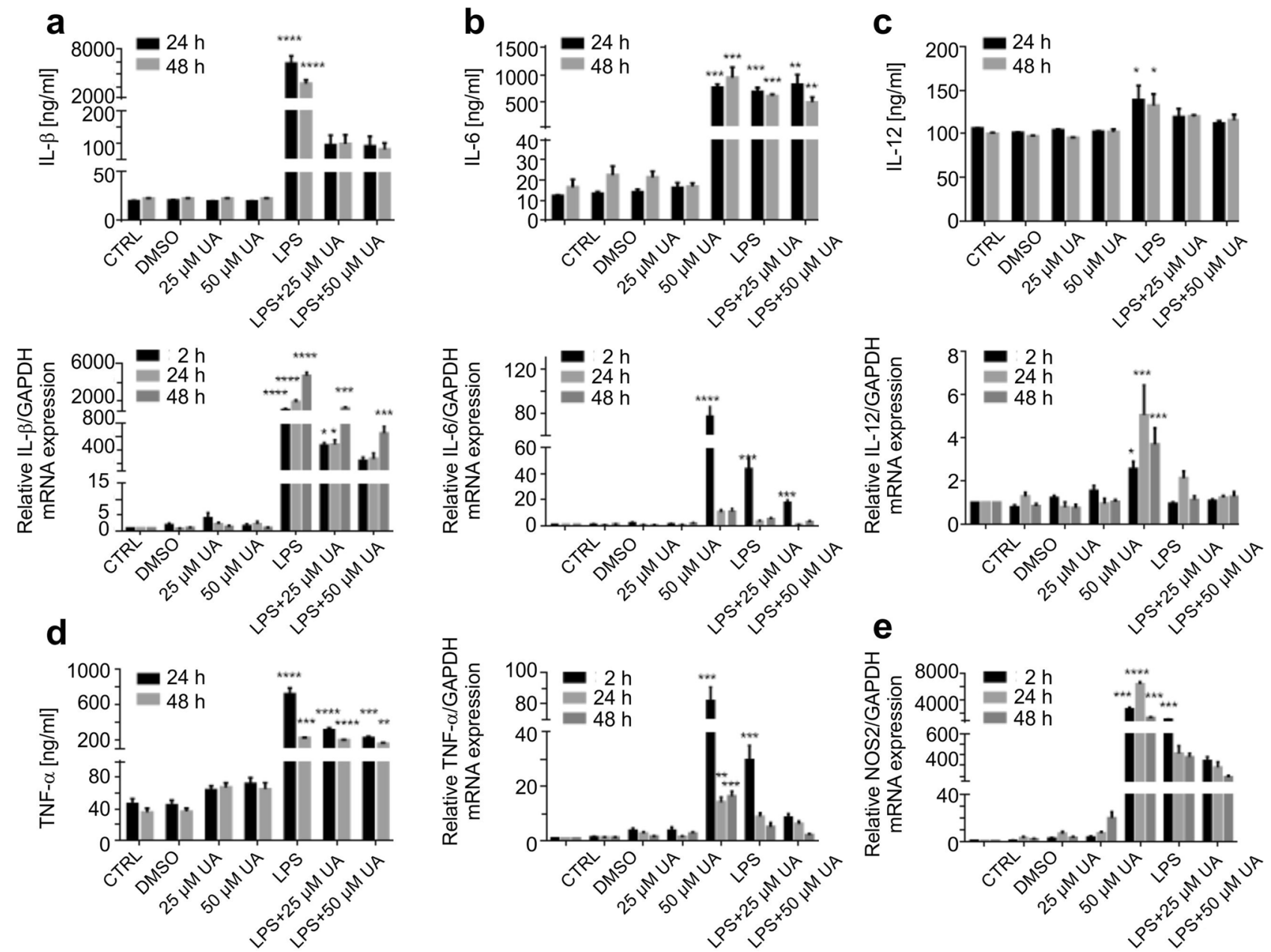

Figure 4. Urolithin A reduced the pro-inflammatory cytokine production and mRNA expression in LPSstimulated murine BMDMs. Murine BMDMs were stimulated by $1 \mu \mathrm{g} / \mathrm{ml}$ of LPS in the presence or absence of UA $(25 \mu \mathrm{M}$ or $50 \mu \mathrm{M})$. Subsequently, expression of the pro-inflammatory cytokines IL-1 $\beta$ (a), IL-6 (b), IL-12 (c), TNF- $\alpha(\mathbf{d})$, and NOS2 (e) were measured in BMDMs by qRT-PCR (over GAPDH) and ELISA at depicted time points. The unstimulated and untreated BMDMs were used as control. BMDMs treated with DMSO were used as negative control. Arithmetic means \pm SEM from seven independent experiments are depicted. Two way ANOVA was used and ${ }^{\star}(p<0.05),{ }^{* *}(P 0.01),{ }^{* * *}(p<0.001)$, and ${ }^{* * * *}(p<0.0001)$ indicate statistically significant differences compared to respective control. Abbreviations: DMSO, dimethyl sulfoxide; UA, urolithin A; LPS, lipopolysaccharides; IL, Interleukin; GAPDH, glyceraldehyde 3-phosphate dehydrogenase; and TNF, tumor necrosis factor; NOS, nitric oxide synthase.

Urolithin A blunted MAPK activation in LPS-stimulated murine BMDMs. The effect of UA on MAPK involved in the regulation of TLR4 expression was investigated after LPS-stimulation in murine BMDMs. Total and phosphorylated ERK1/2 (Fig. 5a, c), p38 (Fig. 6a, b), and SAPK/JNK (Fig. 6a, c) were analysed by immunoblot and normalized to GAPDH at the depicted time intervals. UA alone did not significantly modify total p38 and SAPK/JNK expression, but significantly upregulated total ERK1/2. The administration of UA $(25 \mu \mathrm{M}$ or $50 \mu \mathrm{M})$ to LPS-stimulated BMDMs induced a dose dependent decrease in p38, and SAPK/JNK phosphorylation and had no effect on ERK1/2 phosphorylation (Fig. 5). A representative image of protein expression at $2 \mathrm{~h}$ and $72 \mathrm{~h}$ is shown in Supplementary Fig. 5.

Urolithin A suppressed AKT and mTOR stimulation induced by LPS-stimulation in murine BMDMs. Several TLRs induce the PI3K/Akt pathway which can regulate the immune response in a negative or positive manner ${ }^{39}$. Both Akt and mTOR are principal signalling pathways that orchestrate the response of macrophages to various metabolic and inflammatory signals ${ }^{40}$. In order to clarify the mechanisms of TLR4 regulation by LPS and how the gut microbiota metabolite urolithin A can drive these signalling pathways, expression of PI3K/AKT/mTOR was evaluated. As shown in Fig. 7, UA $(25 \mu \mathrm{M}$ or $50 \mu \mathrm{M})$ alone did not significantly modify total AKT expression but significantly increased total mTOR expression. LPS significantly increased both AKT (Fig. 7a, b) and mTOR (Fig. 7a, c) phosphorylation within $2 \mathrm{~h}$ and continued up to $72 \mathrm{~h}$ (Supplementary Fig. 5), an effect dose dependently blunted by UA. 


\section{Discussion}

The obtained results reveal a powerful anti-inflammatory effect of the gut microbiota UA, which is paralleled by suppression of MAPK and PI3K activation and impairment of $\mathrm{IkB} \alpha$ phosphorylation as well as quenched production of pro-inflammatory miRNA, cytokines and mediators in LPS-stimulated BMDMs (Fig. 8). In addition, the anti-oxidant ability of UA manifested by its ability to preserve DNA breaks through abolishing the cellular ROS production and suppressing calcium entry in LPS-stimulated BMDMs.

TLRs play essential roles in triggering innate immune responses against bacteria and viruses ${ }^{41,42}$. TLR4, which recognizes LPS, is highly expressed by macrophages, dendritic cells, and monocytes ${ }^{43}$. Activation of TLR4-dependent signalling processes in macrophages was estimated with different methods, especially I $\mathrm{B} \alpha$ degradation and NF- $\mathrm{kB}$ phosphorylation and nuclear translocation followed by a series of intracellular signaling pathways, which finally leads to the transcription/translation of inflammatory cytokines ${ }^{44}$. Our data revealed that the treatment with UA induced remarkable changes on LPS-stimulated BMDMs. In order to elucidate the effect of UA on TLR4 expression and the underlying molecular mechanism by which UA exerts anti-inflammatory activity, its influence on the activation of the NF- $\kappa \mathrm{B}$, MAPK and PI3K pathways were evaluated in BMDMs challenged with LPS. Once LPS ligate to TLR4, a series of intracellular signaling pathways are activated, which ultimately lead to the transcription/translation of inflammatory molecules ${ }^{45,46}$. As shown in Fig. 5, UA inhibits the phosphorylation of I $\kappa \mathrm{Ba}$ and prevents the nuclear translocation of NF- $\kappa \mathrm{B}$. Such impairment of NF- $\kappa \mathrm{B}$ activation may be due to the suppressive effect of UA against IL-1 $\beta$, IL-2, IL-6, IL-12, TNF $\alpha$ and NOS2 (Fig. 4). In addition, both MAPK (Figs. 5, 6) and PI3K (Fig. 7) pathways are also stimulated by LPS and mediate the transcription of various miRNA, cytokines and chemokines ${ }^{47,48}$. Our results revealed that UA suppressed the phosphorylation of MAPK (p38 and SAPK/JNK) (Figs. 5, 6), AKT and mTOR (Fig. 7). These results suggest that these two signaling pathways may be possible targets of UA and could mediate the reduction of NOS2, IL-1 $\beta$, IL-2, IL-6, IL-12 and TNFa levels.

The consumption of several natural plant products such as pomegranates, walnuts and berries results in the production of high levels of polyphenolic compounds, specifically ellagitannins and ellagic acid ${ }^{12,13}$. Acid hydrolysis of ellagitannins releases free ellagic acid $(\mathrm{EA})^{14}$. The absorption of ellagitannins and ellagic acid is very low and so, the unabsorbed compounds are further metabolised by gut microbiota to bioactive molecules including the different urolithin compounds A, B, C, and $\mathrm{D}^{14,17}$. Among urolithins, urolithin A (UA; 3,8-dihydroxybenzo[c] chromen-6-one) is the most relevant urolithin and has been shown to influence the microbiota composition in rat models ${ }^{49}$, but the significance of these changes remain to be established. The gut bacterial metabolites such as SCFAs including propionic acid, acetic acid, and butyric acid can positively contribute to activation of the adaptive immunity ${ }^{20,23,50}$.

UA has an anti-inflammatory property in inflammatory bowel disease and improves the gut permeability ${ }^{51}$. It has also been observed that UA may play an important role in the inhibition of certain cancers, such as colorectal or prostate cancers ${ }^{52,53}$. In addition to its anti-inflammatory activity, UA preserves gut barrier integrity by increasing tight junction proteins induced by activation of aryl hydrocarbon receptor through the NF-E2 p45-related factor 2 (NRF2)-dependent pathway ${ }^{49,54}$.

MicroRNAs are involved in all facets of immune system development starting from hematopoiesis to activation during the immune response to harmful stimuli ${ }^{55}$. Dysregulation of miRNA can induce immune disturbance leading to various diseases including cancer $^{56}$. LPS is a potent stimulator of several miRNA including miR-146a, miR-132 and miR-155 57 .

Promoter analysis studies recognized miR-146a as NF- $\kappa B$-inducible miRNA. Also, the study identified a crucial role of miR-146 in controlling TLR and cytokine signalling via a negative feedback regulation loop ${ }^{57}$. Our results show that UA was able to supress the expression level of miR-10, miR-99b, miR146a and miR-155 after LPS-stimulation. On the contrary, Bazzoni, Rossato et al.$^{58}$ reported that miR-9 is activated by TLR4 in human PMNs and monocytes targeting NF- $\mathrm{BB}$ subunit $\mathrm{p} 50$. Therefore, suppressing the production of a proinflammatory cytokine as inhibition of NF- $\kappa B$ and MAPK activation may influence or mediate the reduction of miRNA expression.

Functional properties of the immune cells, including activation, chemokine and cytokine expression are further regulated by $\mathrm{Ca}^{2+}$ signalling ${ }^{59,60}$. Our data revealed that UA abolished the upregulation of intracellular calcium levels induced by LPS stimulation. Such inhibition may be achieved through the modulation of IP3R activity where UA was able to decrease the AKT phosphorylation. In parallel, UA quenched the elevation in mitochondrial and cellular ROS production induced by LPS stimulation. UA treatment apparently blocked ROS generation and restored the balance of the intracellular redox state by attenuating oxidative stress. In agreement with previous reports ${ }^{61,62}$, our results show that excessive ROS production induced by LPS stimulates MAPK and NF- $\kappa B$ pathways and initiates inflammatory responses. In addition, our data revealed that excessive ROS production and calcium entry induced by LPS stimulate PI3K/AKT/mTOR pathways. Such decrease in ROS production and entry of calcium was concomitant with prominent reduction in DNA double strand breaks induced by LPS stimulation after $48 \mathrm{~h}$. This result was in agreement with the findings of Chumduri, Gurumurthy et al., who indicated that LPS can induce DSBs either directly or indirectly through reactive oxygen species ${ }^{63}$. In addition, Qiao, Huang et al. revealed that LPS-induced DSBs activated the NF- $\kappa$ B signalling pathway in human dental pulp tissues ${ }^{64}$.

Taken together, the results presented in this study demonstrate that UA differentially modulated the production of inflammatory miRNA and cytokines and suppressed LPS-induced effects on NOS2, ROS production, intracellular calcium, and DNA double strand breaks. These effects are most likely mediated by the suppression of IкB $\alpha$ phosphorylation, MAPK (p38 and JNK) and PI3K/AKT/mTOR signaling pathways (Fig. 8). Our findings highlight the potential of UA as a novel therapeutic agent for treating inflammatory diseases such as IBD. Further in vivo studies are essential to confirm the therapeutic or preventive effect of UA in animal disease models. 
a
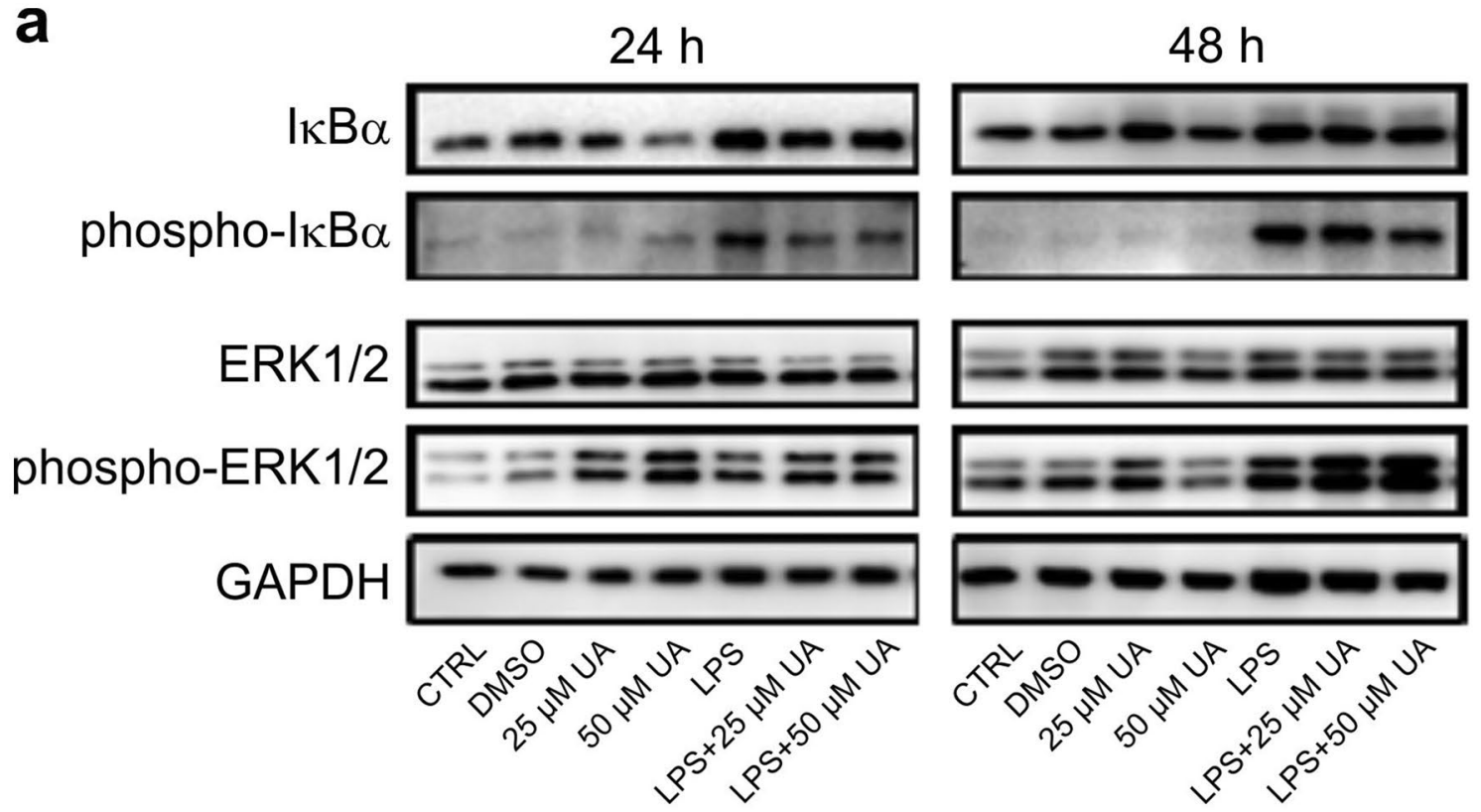

b
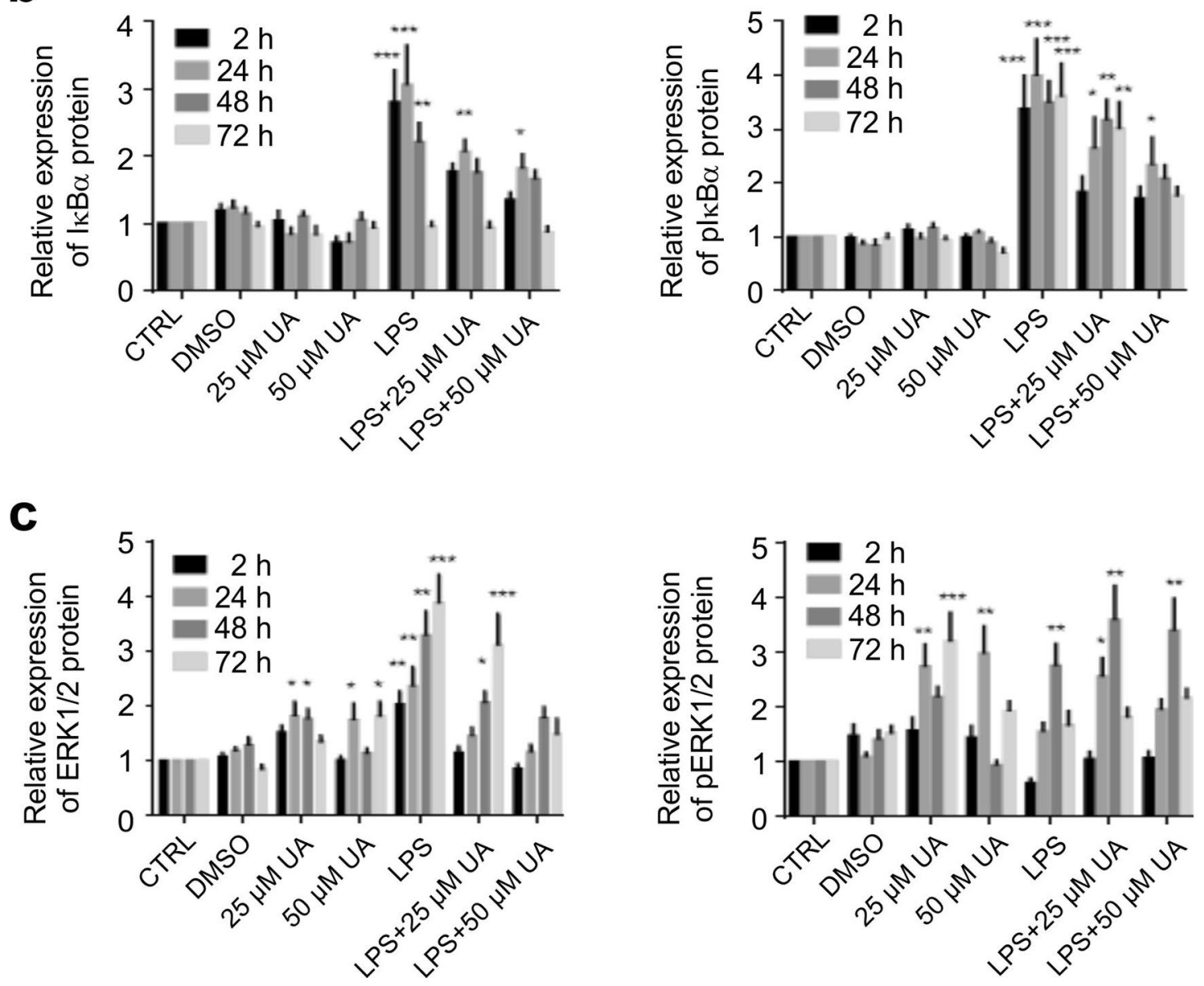


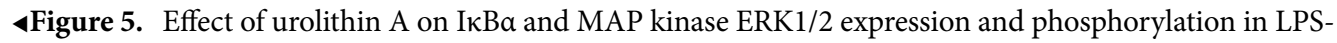
stimulated murine BMDMs. Murine BMDMs were stimulated by $1 \mu \mathrm{g} / \mathrm{ml}$ of LPS in the presence or absence of UA $(25 \mu \mathrm{M}$ or $50 \mu \mathrm{M})$ and harvested at indicated time intervals, followed by western blot analysis.

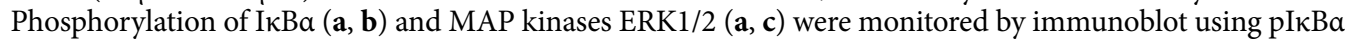
(Ser32/36) monoclonal antibodies and phospho-p44/42 MAP kinase (Thr202/Tyr204) polyclonal antibodies, respectively at depicted time points. Subsequently, blots were stripped and re-incubated with antibodies against total IкBa and ERK1/2. GAPDH served as a loading control. The unstimulated and untreated BMDMs were used as control. BMDMs treated with DMSO were used as negative control. Representative images and arithmetic means \pm SEM from five independent experiments are depicted. Two way ANOVA was used and ${ }^{\star}(p<0.05),{ }^{* *}(P 0.01)$, and ${ }^{* * *}(p<0.001)$ indicate statistically significant differences compared to respective control. Full length blots are found in the Supplementary File 9. Abbreviations: DMSO, dimethyl sulfoxide; LPS, lipopolysaccharides; UA, urolithin A; GAPDH, glyceraldehyde-3-phosphate dehydrogenase.

\section{Materials and methods}

Mice. C57BL/6 J mice between 8-12 weeks of age (both male and female) were used for experiments. All experiments were performed according to the EU Animals Scientific Procedures Act and the German law for the welfare of animals. All procedures were approved by the authorities of the state of Baden-Württemberg, i.e. Regierungspräsidium Tübingen.

Bone marrow cells isolation, culture and treatment. Bone marrow cells (BMCs) isolated from femurs and tibias under sterile conditions were cultured in DMEM media (\#61965-026- Life Technologies, Germany), supplemented with 10\% FBS (\#10270-106 - Life Technologies, Germany), 100 U/ml penicillin/streptomycin (\#P4333- Sigma-Aldrich, Germany), 10\% MEM Non-essential Amino Acid Solution (\#M7145- Sigma-Aldrich, Germany), 0.1\% 2-Mercaptoethanol (\#M7522- Sigma-Aldrich, Germany) and 20\% (v/v) L929 cell-condition medium (LCM) as a source of M-CSF. After 7 days in culture, adherent bone marrow-derived macrophages (BMDMs) were more than $90 \%$ pure. The efficiency of the differentiation is assessed using FACS analysis surface antigen expression. BMDMs were stimulated with $1 \mu \mathrm{g} / \mathrm{ml}$ LPS (Escherichia coli O111:B4, \#L4391, SigmaAldrich, Germany) in the presence or absence of $(25 \mu \mathrm{M}$ or $50 \mu \mathrm{M})$ urolithin A (UA, \#1143-70-0, Santa Cruz, Germany) for $2 \mathrm{~h}, 24 \mathrm{~h}, 48 \mathrm{~h}$ and $72 \mathrm{~h}$. The untreated and unstimulated BMDMs were used as control and those received Dimethyl sulfoxide (DMSO - \#A994-2, Roth, Germany) only were used as negative control.

Phenotypic characterization of BMMs with directly conjugated cell surface antibodies. BMMs were detached by Accutase (\#SCR005, Sigma-Aldrich) and collected in a 96 well plate, washed with $1 \times$ PBS (\#D8537, Sigma Aldrich, Germany), then centrifuged and re-suspended in $50 \mu \mathrm{l}$ of fresh $1 \times \mathrm{PBS}$. After that, $0.5 \mu \mathrm{l}$ fluorescently-labelled-antibodies CD11b-PE (\#12-0112-82), F4/80-FITC (\#11-4801-81) and MHCII-APC (\#17-5321-82) were added to each well. Cells were incubated in the dark for $30-45 \mathrm{~min}$ at $4{ }^{\circ} \mathrm{C}$. After incubation, BMDMs were washed and acquired on the flow cytometry- BD FACS Calibur ${ }^{\text {rix }}$ (BD Bioscience, Germany). The harvested BMCs (20.000 cells) were relatively favored to differentiate into macrophages (Supplementary Fig. 6) with CD $11 b^{\text {high }}$ (almost $\left.100 \%\right), \mathrm{F} 4 / 80^{\text {high }}(90 \% \pm 7 \%)$ and MHCII ${ }^{\text {low }}(10 \% \pm 4 \%)$. In addition, the second characterization after LPS-stimulation in presence or absence of UA revealed that all groups are still F4/80 high, $\mathrm{CD} 11 \mathrm{~b}^{\text {high }}$ and MHCII ${ }^{\text {low }}$ (Supplementary Fig. 7). The data were analysed by FlowJo software (FLOWJO, LLC, USA).

Influence of urolithin A on the viability of murine BMDMs. BMDM (20.000 cells) were isolated, stimulated and treated as described above. The percentage of apoptotic cells was estimated by flow cytometry using the AnnexinV apoptosis detection kit FITC (\#88-8005- 72, eBioscience) in accordance with the manufacturer's instructions. Briefly, BMDMs were collected, washed and re-suspended in $1 \times$ binding buffer containing Annexin V-FITC solution (1:50 dilution). After that, BMDMs (20.000 cells) were incubated at room temperature for $15 \mathrm{~min}$, protected from light, and washed again. After adding Propidium Iodide solution (1:100 dilution), BMDMs were incubated at room temperature in the dark for $10 \mathrm{~min}$ prior to flow cytometry for cell apoptosis analysis $^{65,66}$. The observed data show that more than $95 \%$ of the untreated control group were viable and nonapoptotic (Annexin $\mathrm{V}^{-} \mathrm{PI}^{-}$). Dot plots show the effects of UA on LPS-stimulated BMDMs after $72 \mathrm{~h}$ (Supplementary Fig. 8a, c). BMDMs were treated with varying concentration of UA (for $72 \mathrm{~h}$ ) ranging from 10-300 $\mu \mathrm{M}$ to demonstrate the dose dependent effect on apoptosis (Supplementary Fig. 8B). The apoptotic effects start to appear at $100 \mu \mathrm{M}$ and according to these results, $25 \mu \mathrm{M}$ UA and $50 \mu \mathrm{M}$ UA were used for further experiments. Data were analyzed by Flowjo software (FLOWJO, LLC, USA).

Measurements of intracellular calcium and reactive oxygen species(ROS) production. BMDMs were treated as desired and then collected and washed once and re-suspended in 96 well plates with $200 \mu \mathrm{l}$ of $1 \times$ PBS. In the following, $1 \mu \mathrm{M}$ Fluo-4 (\#F14200, Invitrogen, Germany) or $10 \mathrm{nM}$ of ROS sensitive dye 2',7'-Dichlorofluorescin diacetate (H2DCFDA, \#D6883, Sigma-Aldrich, Germany) were added. BMDMs were incubated for $30 \mathrm{~min}$ at $37^{\circ} \mathrm{C}$ in the dark and then washed twice with $1 \times$ PBS buffer. Finally, BMDMs were re-suspended in $200 \mu \mathrm{l}$ of PBS prior to flow cytometry (FL1 channel) for measurement with a FACSCalibur ${ }^{\mathrm{Tn}}$. 20,000 cells/sample in the flow cytometer were acquired using the recommended software for data acquisition. Obtained values were corrected for autofluorescene of control cells without dye ${ }^{66,67}$. Geometric mean of the FL-1 signal intensity was used to show the amount of Fluo-4 and H2DCFDA fluorescence intensity (separate measurements). Data were analyzed by FlowJo software (FLOWJO, LLC, USA). 
a

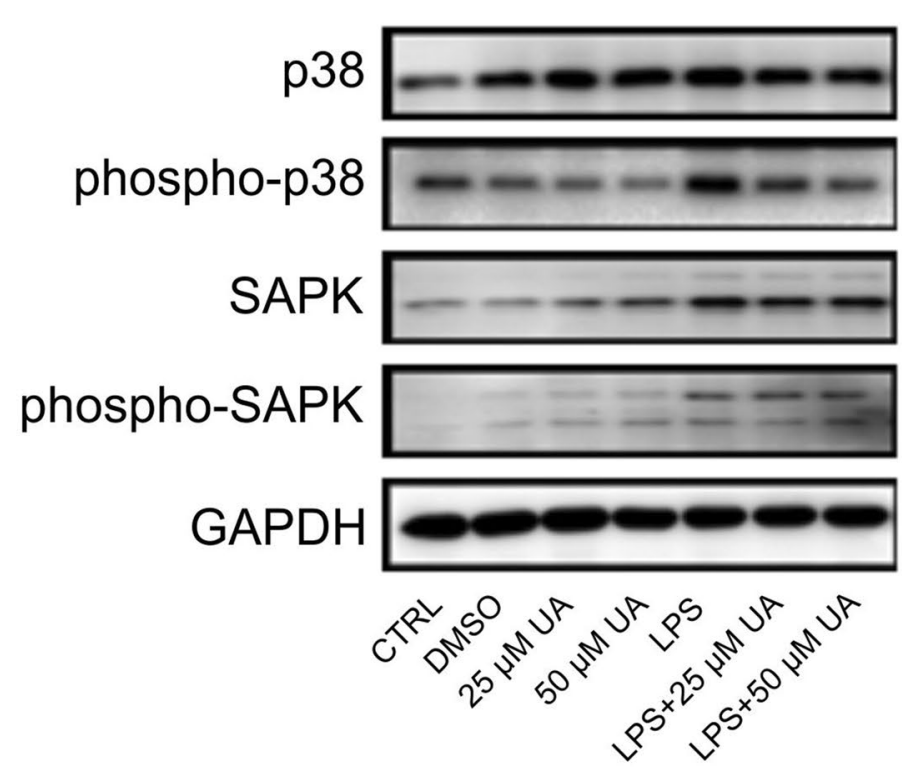

$48 \mathrm{~h}$
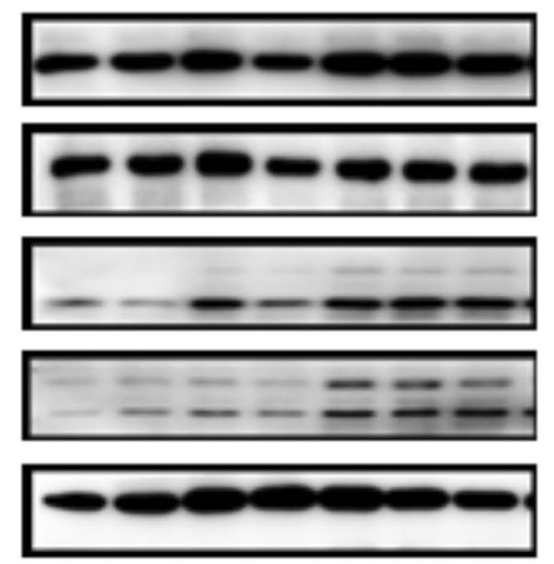

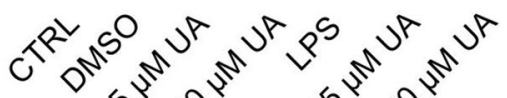

$b_{2} 0^{2}$ $b^{x^{x^{2}}} 5^{s^{x^{2}}}$

b
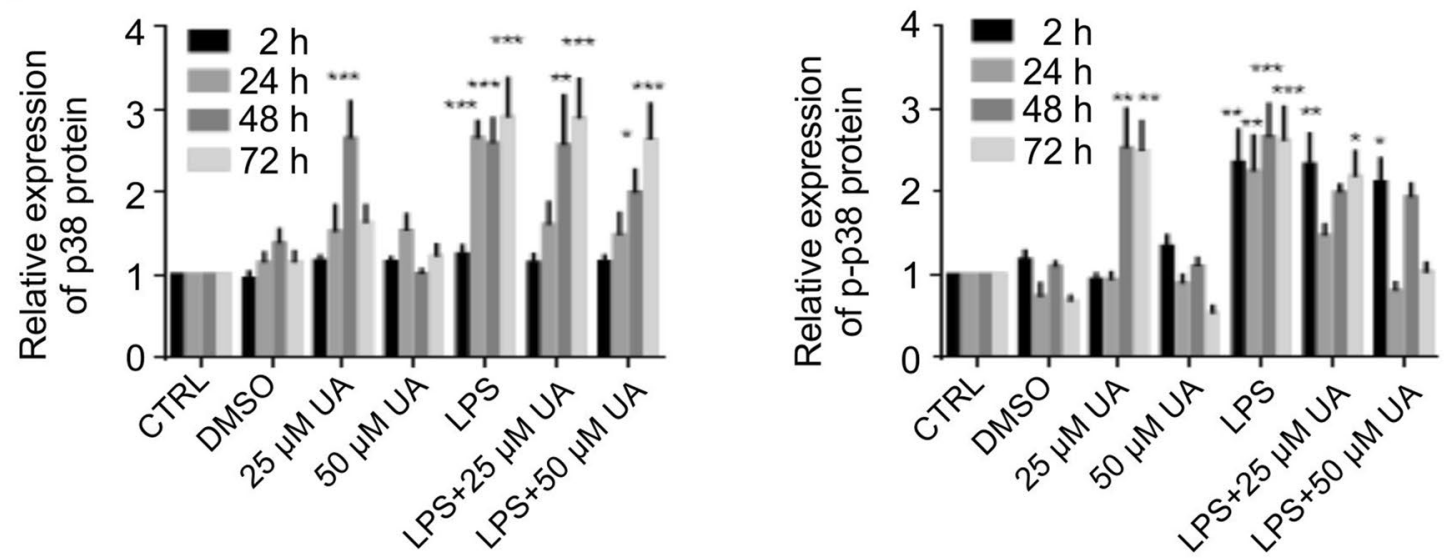

C
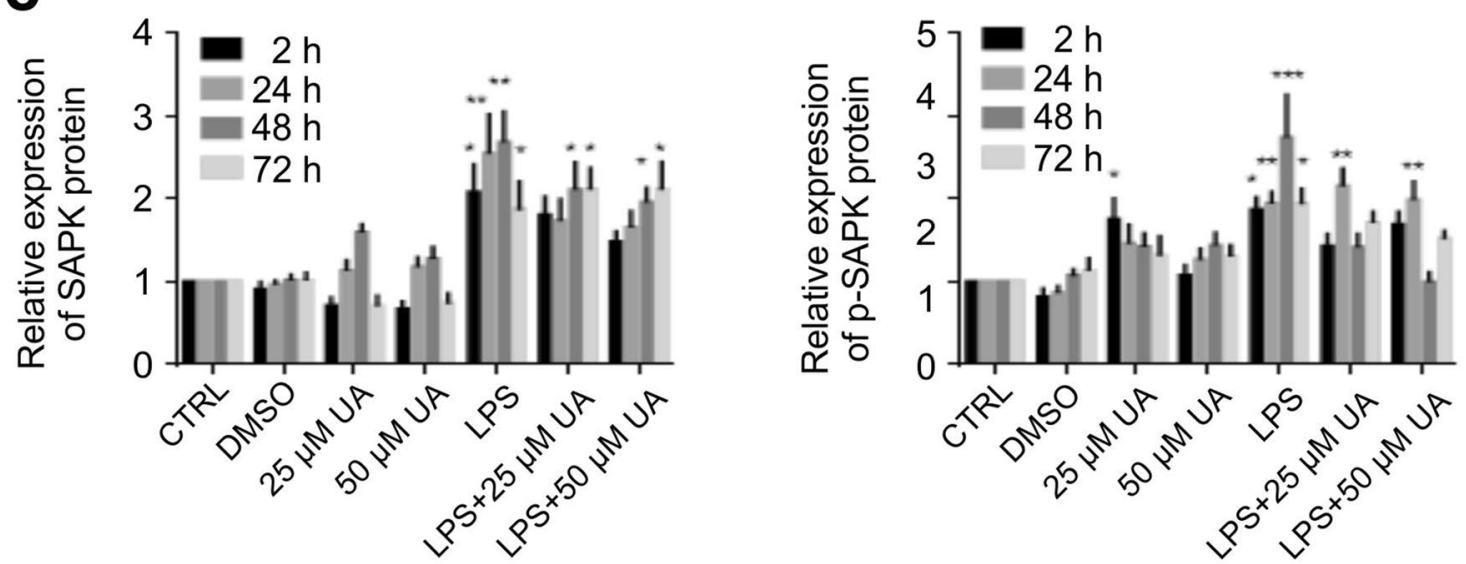
4Figure 6. Effect of urolithin A on MAP kinase p38 and SAPK/JNK expression and phosphorylation in LPS-stimulated murine BMDMs. Murine BMDMs were stimulated by $1 \mu \mathrm{g} / \mathrm{ml}$ of LPS in the presence or absence of UA $(25 \mu \mathrm{M}$ or $50 \mu \mathrm{M})$ and harvested at indicated time intervals, followed by western blot analysis. Phosphorylation of MAP kinase p38 (a, b), and SAPK/JNK (a, c) were monitored by immunoblot using phospho-p38 MAP kinase (Thr180/Tyr182) and phospho-SAPK/JNK MAP kinase (Thr183/Tyr185) monoclonal antibodies, respectively at depicted time points. Subsequently, blots were stripped and re-incubated with antibodies against total p38 and SAPK/JNK. GAPDH served as a loading control. The unstimulated and untreated BMDMs were used as control. BMDMs treated with DMSO were used as negative control. Representative images and arithmetic means \pm SEM from five independent experiments are depicted. Two way ANOVA was used and ${ }^{\star}(p<0.05),{ }^{* *}(P 0.01)$, and ${ }^{* * *}(p<0.001)$ indicate statistically significant differences compared to respective control. Full length blots are found in the Supplementary File 9. Abbreviations: DMSO, dimethyl sulfoxide; LPS, lipopolysaccharides; UA, urolithin A; GAPDH, glyceraldehyde-3- phosphate dehydrogenase.

MitoSOX Red mitochondrial superoxide indicator. BMDMs (10.000 cells per well) were grown, stimulated as indicated in X-well tissue culture chamber (\#94.6150.401, Sarstedt, Germany), and further incubated for $48 \mathrm{~h}$. BMDMs were washed with PBS and subsequently incubated for $10 \mathrm{~min}$ at $37^{\circ} \mathrm{C}$ in $500 \mu \mathrm{l}$ of $5 \mu \mathrm{M}$ MitoSOX red reagent (\#M36008D, Thermo Fisher Scientific, USA) protected from light. BMDMs were washed three times and one drop of ProLong GOLD Anti-fade Mountant with DAPI (\#P36931, Thermo Fisher Scientific, USA) was added per well. The fluorescence imaging was performed with an Axiophot Zeiss microscope using a digital camera with AxioVision 4.8 software. The obtained images were analysed by Fiji ImageJ software.

YH2AX for DNA double strand breaks. BMDMs (10.000 cells per well) were seeded on a X-well tissue culture chamber, treated as indicated and further incubated for $2 \mathrm{~h}$ and $48 \mathrm{~h}$. BMDMs were washed with $500 \mu \mathrm{l}$ of PBS per well (using orbital mixer for $5 \mathrm{~min}$ ) then fixed with $4 \%(\mathrm{v} / \mathrm{v})$ formaldehyde (\#158127, Sigma-Aldrich, Germany) for $10 \mathrm{~min}$ at RT, permeabilized for $10 \mathrm{~min}$ at RT with $0.1 \%$ (v/v) Triton-X 100 (\#T9284, SigmaAldrich), then washed with PBS. Non-specific protein binding was blocked with $1 \%$ (v/v) Bovine serum albumin (BSA, \#A2153, Sigma-Aldrich, Germany) for $20 \mathrm{~min}$ at RT. BMDMs were incubated with primary mouse polyclonal anti-histone H2A.X (\#PA5-28778, Thermo Fisher Scientific, USA) antibody for $1 \mathrm{~h}$ at RT in 1:500 dilutions. BMDMs were washed and incubated with Alexa Fluor 488 goat anti-rabbit IgG (\#A-11008, Thermo Fisher Scientific, USA) diluted 1:500 in 1\% BSA at RT for $45 \mathrm{~min}$ in the dark. BMDMs were mounted with ProLong GOLD Anti-fade containing 4',6-diamidino-2-phenylindole (DAPI). The slides were kept in the dark for 30 min at RT before sealing with nail polish and stored overnight at $4{ }^{\circ} \mathrm{C}$ in dark before analysis. The fluorescence imaging was performed with an Axiophot Zeiss microscope using a digital camera with AxioVision 4.8 software. The obtained images were analysed by Fiji ImageJ software.

mRNA and miRNA qRT-PCR. Total RNA including miRNAs was extracted from BMDMs using miRNAeasy Kit (\#217004, Qiagen, Germany). The mRNA $(2 \mu \mathrm{g})$ and miRNAs (100 $\mathrm{ng})$ were separately reverse transcribed using Superscript III First-Strand synthesis system (\#18080-51, Invitrogen, Germany) and miRNA universal cDNA synthesis kit II (\#203301, Exiqon, Denmark) for reverse transcript PCR (RT-PCR) and subsequent real-time quantitative PCR (qRT-PCR). Detection of gene expression was performed with KapaFast-SYBR Green (\#KAPBKK4606, Peqlab, Germany) and measurements were performed on a BioRad iCycler iQ RealTime PCR Detection System (Bio-Rad Laboratories). The relative expression levels of mRNAs and miRNA were normalized to that of GAPDH and $5 S$ rRNA, respectively ${ }^{68}$. The murine primers used to detect IL-1 $\beta$, IL-2, IL4, IL-6, IL-10, IL-12, TNFa, IFN $\gamma$, TGF- $\beta$, and NOS2 expression are summarized in Table 1. For amplification of different miRNAs, hsa-miR-9-5p LNA PCR primer set (\#204513), hsa-miR-10a-5p LNA TM PCR primer set (\#204778), has-miR-99b-5p LNA PCR primer set (\#205983), hsa-miR-146a-5p LNA PCR primer set (\#204688), mmu-miR-155-5p LNA PCR primer set (\#205930), and reference 5S rRNA primer set (\#203906) were used and the reaction was set up as recommended by Exiqon ${ }^{67,69}$.

Immunoblotting. BMDMs were stimulated and treated as indicated then washed once with PBS (\#D8537, Sigma-Aldrich, Germany) and equal amounts of $\mathrm{H}_{2} \mathrm{O}$ and 2X Lammelli's Buffer for cell lysis were added. Proteins were denatured at $95^{\circ} \mathrm{C}$ for $7 \mathrm{~min}$ and stored at $-20^{\circ} \mathrm{C}$. Equal amounts of proteins $(15 \mu \mathrm{g})$ were separated on $10 \%$ sodium dodecyl sulfate-polyacrylamide (SDS) gels and transferred to PVDF membranes (\#10600023, Amersham Biosciences, UK). Nonspecific binding sites were blocked for $1 \mathrm{~h}$ at room temperature with 5\% nonfat dry milk in Tris-buffered saline with $1 \%$ Tween. Membranes were probed with the indicated primary antibodies (all from Cell Signaling Technology and diluted 1:1000): anti- TLR2 (\#13744s), TLR4 (\#14358s), ERK1/2 (\#9102s), pERK1/2 (Thr202/Tyr204, \#9101s), SAPK/JNK (\#9252s), pSAPK/JNK (Thr183/Tyr185, \#4671s), p38 (\#8690S), pp38 (Thr180/Tyr182, \#4511s), IкBa (\#4812s), pIкBa (Ser32, \#2859S), AKT (\#9272s), pAKT (T308, \#2965s), mTOR (\#2983s), phospho-mTOR (Ser2448, 2971s), and GAPDH (1:2000; \#2118s) followed by HRPconjugated secondary antibodies (\#7074P2). Membranes were washed thrice and the antibodies visualized with enhanced chemiluminescent HRP substrate (\#R-03031-D25 and R-03025-D25, advansta, USA). For detection of signals, X-ray films or versa doc were used. To confirm loading control after detection of phosphorylated protein, membranes were stripped using ReBlot Plus strong antibody stripping solution (\#2504, Merck, Germany), blocked and re-probed with different antibodies against whole protein. Protein bands were quantified using Image lab \& ImageJ software. Results are shown as the ratio of total protein to GAPDH normalized to 
a

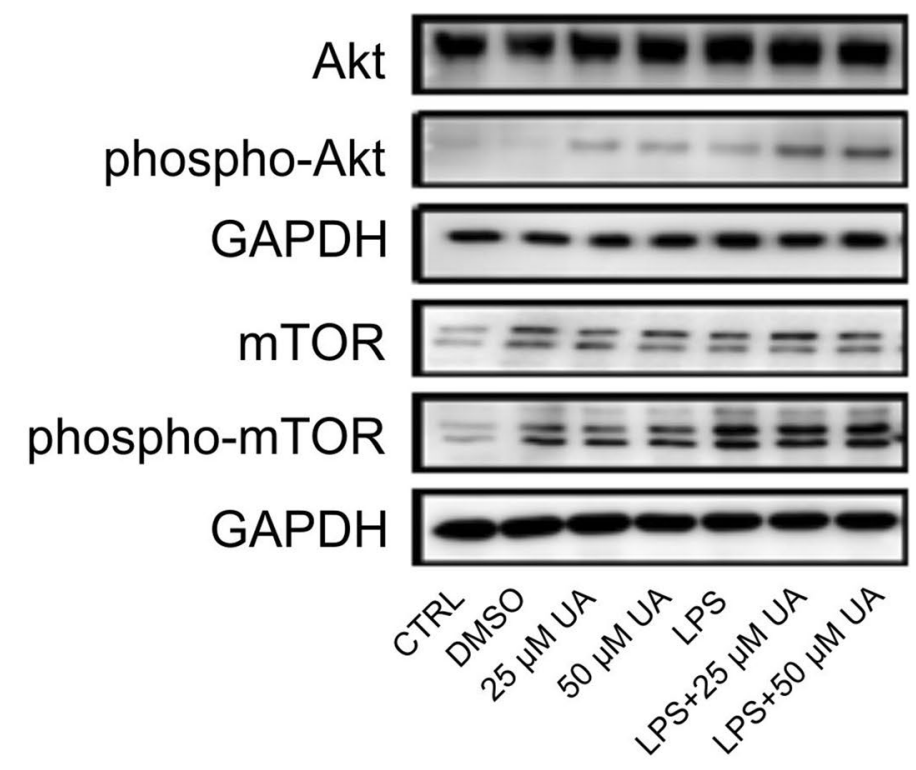

$48 \mathrm{~h}$

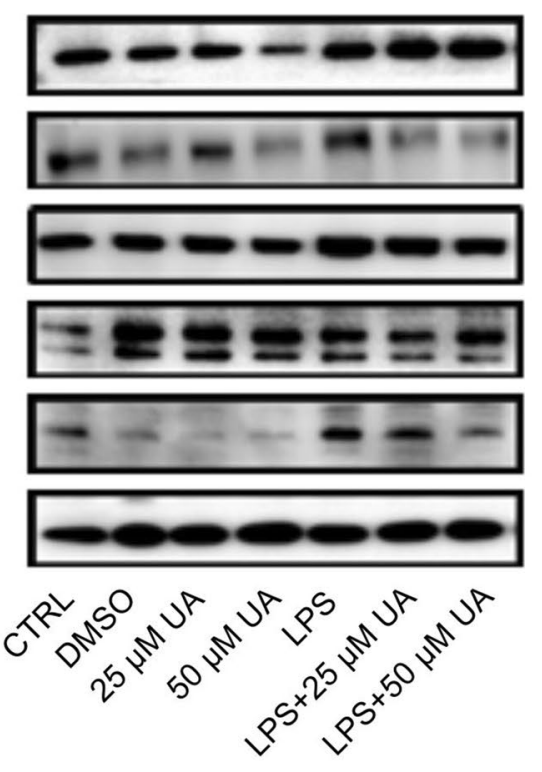

b
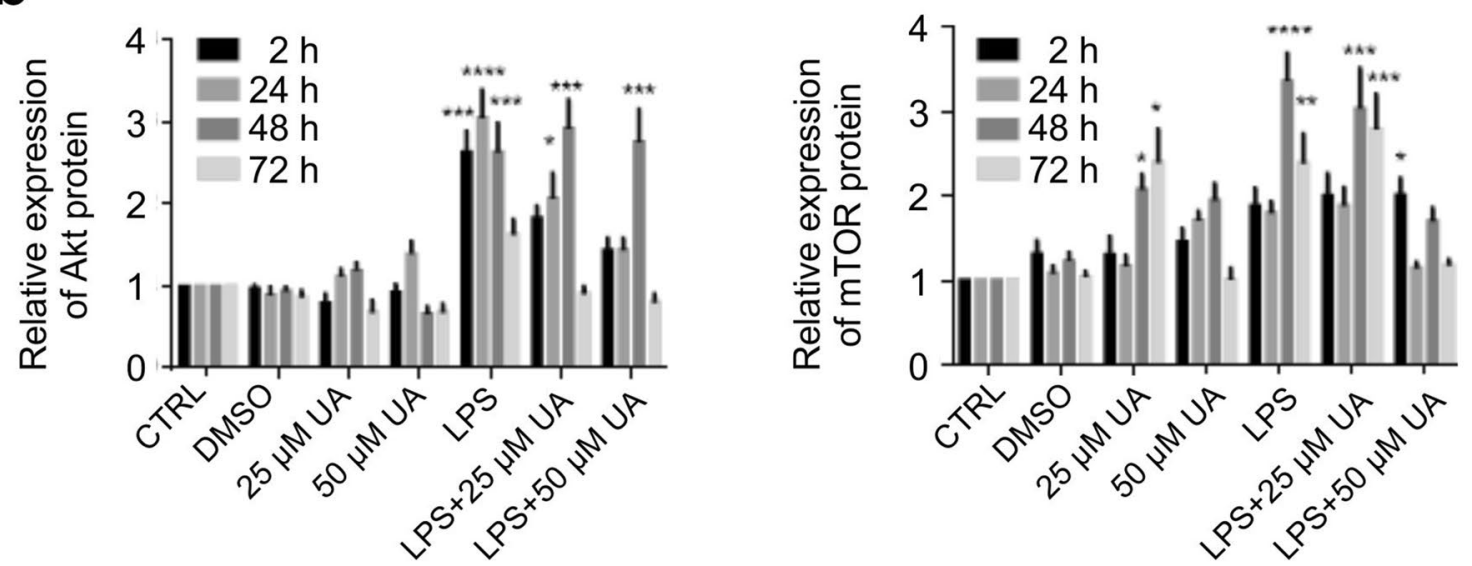

C
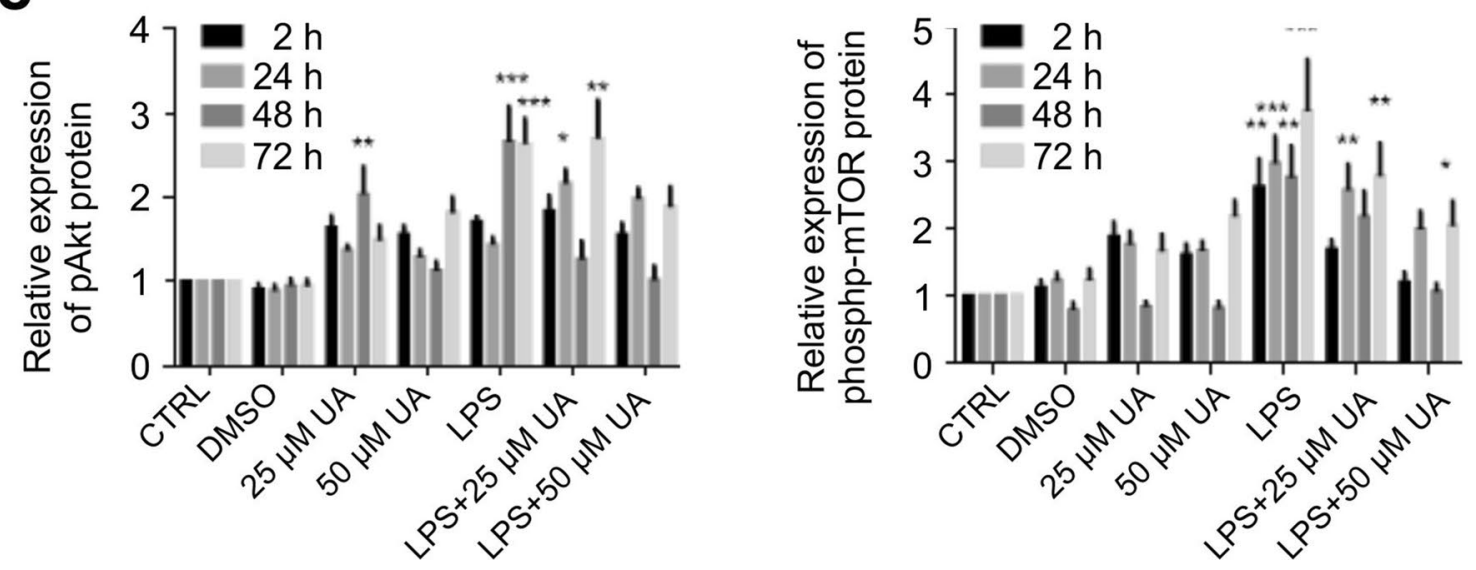
4Figure 7. Urolithin A diminished PI3K/AKT/mTOR activation in LPS-stimulated murine BMDMs. Murine BMDMs were stimulated by $1 \mu \mathrm{g} / \mathrm{ml}$ of LPS in the presence or absence of UA $(25 \mu \mathrm{M}$ or $50 \mu \mathrm{M})$ and harvested at indicated time intervals followed by western blot analysis. Phosphorylation of AKT (a, b) and mTOR (a, c) were monitored by immunoblot using pAKT (Ser473) and phospho-mTOR (Ser2448) monoclonal antibodies. Subsequently, blots were stripped and re-incubated with antibody against total AKT and mTOR. GAPDH served as a loading control. The unstimulated and untreated BMDMs were used as control. BMDMs treated with DMSO were used as negative control. Representative images and arithmetic means \pm SEM from five independent experiments are depicted. Two way ANOVA was used and ${ }^{\star}(p<0.05),{ }^{\star *}(P 0.01),{ }^{\star * *}(p<0.001)$, and ${ }^{* * * *}(p<0.0001)$ indicate statistically significant differences compared to respective control. Full length blots are found in the Supplementary File 9. Abbreviations: DMSO, dimethyl sulfoxide; LPS, lipopolysaccharides; UA, urolithin A; GAPDH, glyceraldehyde-3- phosphate dehydrogenase.

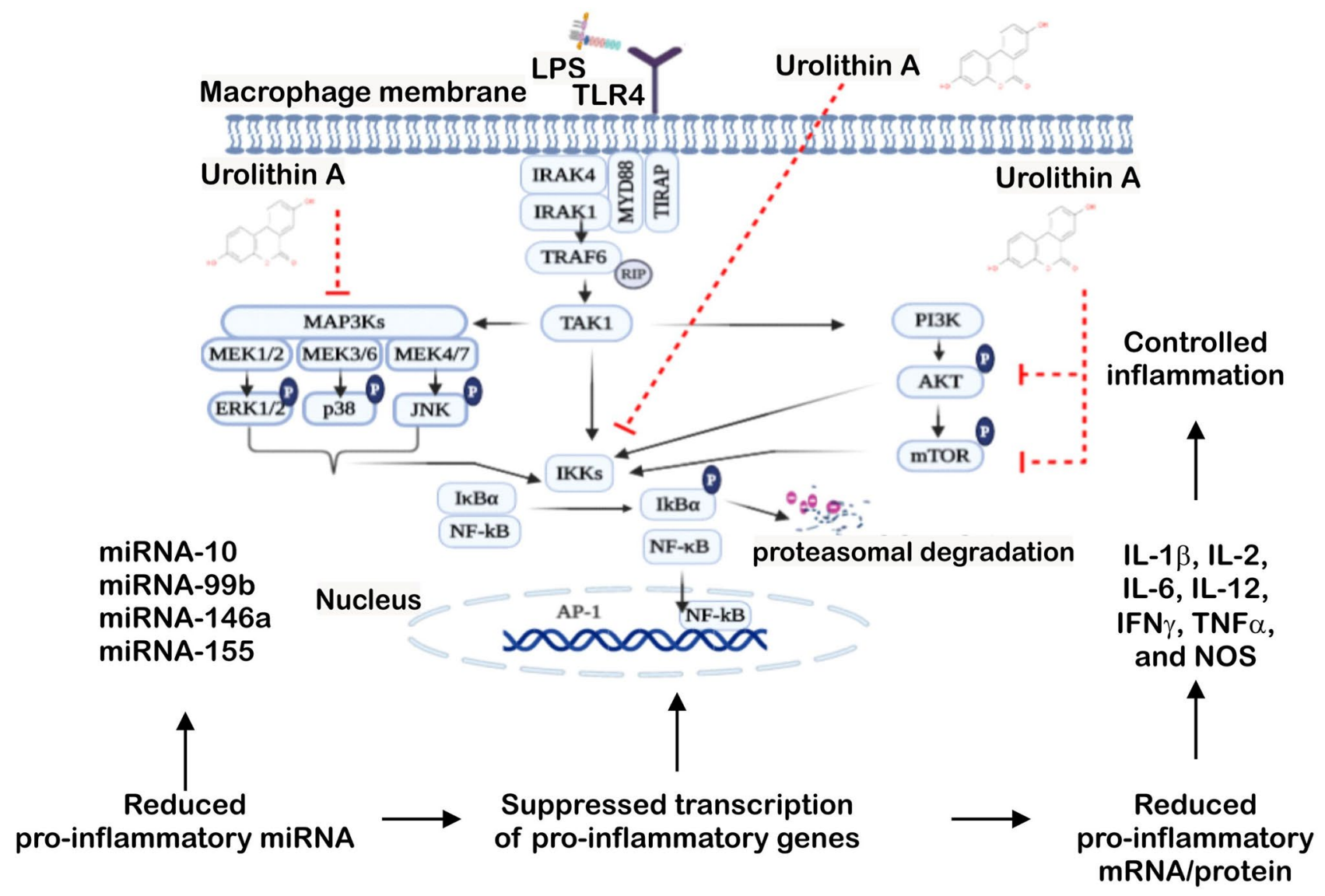

Figure 8. Urolithin A controlled NF-kB inflammatory response. LPS stimulates TLR4 signalling cascades. Urolithin A impairs the NF-kB activation through the inactivation of IKKs. Urolithin A suppresses MAPKs activation through blocking ERK, p38 and JNK phosphorylation. Additionally, urolithin A inhibits the phosphorylation of AKT and mTOR protein. Abbreviation: LPS; lipopolysaccharide; IR, ionizing radiation; TIRAP, TIR adaptor protein; MyD88, myeloid differentiation primary-response gene 88; IRAK, interleukin-1 receptor associated kinase; TRAF, TNF receptor associated factor; RIP, receptor-interacting protein kinases; TAK, transforming growth factor beta-activated kinase 1; IKK, IкB kinase; MAPK, mitogen activated protein kinase; PI3K, phosphatidylinositol 3-kinases; mTOR, mechanistic target of rapamycin; ERK, extracellular regulated kinase; JNK, c-Jun N-terminal kinase; NF- $\kappa \mathrm{B}$, nuclear factor- $\kappa \mathrm{B}$; I $\mathrm{B}$, kinase complex; AP, activator protein; IL, interleukin; IFN, interferon; TNF, tumor necrosis factor; and NOS, nitric oxide.

the untreated control group. To assign the right protein size ProSieve QuadColor Protein Marker was used as a marker (6 $\mu$ l, \#00193837, Lonza).

Enzyme linked immunosorbent assay (ELISA). Supernatants from co-culture assays were analyzed for IL-1 3 (\#88701322), IL-4 (\#88704422), IL-6 (\#88706422), IL-10 (\#88710522), IL-12 (\#88712188), TGF- $\beta$ (\#88835022), TNF- $\alpha$ (\#88732422) and IFN- $\gamma$ (\#88831422) production via sandwich ELISA kits recommended by eBioscience manufacturer's instructions. Cytokines were quantified by comparison to recombinant standard proteins. 


\begin{tabular}{|c|c|}
\hline Primer & Sequences \\
\hline IL- $1 \beta$ & $\begin{array}{l}\text { F 5- TACCTGTGGCCTTGGGCCTCAA - } 3^{-} \\
\text {R 5- } 5^{-} \text {GCTTGGGATCCACACTCTCCAGC - } 3^{-}\end{array}$ \\
\hline IL-2 & $\begin{array}{l}\text { F 5 }^{-} \text {AGGAACCTGAAACTCCCCAG - } 3^{-} \\
\text {R 5- CTTTCAATTCTGTGGCCTGCTT - } 3^{-}\end{array}$ \\
\hline IL-4 & $\begin{array}{l}\text { F }^{-}{ }^{-} \text {AGGAGAAGGGACGCCATGCAC - } 3^{-} \\
\text {R 5 } 5^{-} \text {GCGAAGCACCTTGGAAGCCCTAC - } 3^{-}\end{array}$ \\
\hline IL-6 & $\begin{array}{l}\text { F 5- TGGAGTCACAGAAGGAGTGGCTA - } 3^{-} \\
\text {R 5- TCTGACCACAGTGAGGAATGTCC - 3- }\end{array}$ \\
\hline IL-10 & $\begin{array}{l}\text { F 5- GGCGCTGTCATCGATTTCTCCCC - } 3^{-} \\
\text {R 5- GGCCTTGTAGACACCTTGGTCTT - } 3^{-}\end{array}$ \\
\hline IL-12 & $\begin{array}{l}\text { F 5- AATCAGGGCTGCGAAGGTA - } 3^{-} \\
\text {R 5 }^{-} \text {AGGCCCTGGTTTCTTATCAA - } 3^{-}\end{array}$ \\
\hline TGF- $\beta$ & $\begin{array}{l}\text { F 5- }^{-} \text {GAGCCCGAAGCGGACTACTA - } 3^{-} \\
\text {R 5 }{ }^{-} \text {TGGTTTTCTCATAGATGGCGTTG - } 3^{-}\end{array}$ \\
\hline TNF- $\alpha$ & $\begin{array}{l}\text { F } 5^{-} \text {ATAGCTCCCAGAAAAGCAAGC - } 3^{-} \\
\text {R 5- CACCCCGAAGTTCAGTAGACA - } 3^{-}\end{array}$ \\
\hline IFN- $\gamma$ & $\begin{array}{l}\text { F 5- GGCTGTTACTGCCACGGCACA - } 3^{-} \\
\text {R 5- CACCATCCTTTTGCCAGTTCCTC - } 3^{-}\end{array}$ \\
\hline NOS2 & $\begin{array}{l}\text { F } 5-^{-} \text {AGTCAACTGCAAGAGAACGGA }-3^{-} \\
\text {R } 5^{-} \text {TGAGAACAGCACAAGGGGTT }-3^{-}\end{array}$ \\
\hline
\end{tabular}

Table 1. Murine primer sequences.

Statistics. Given data are provided as means \pm SEM. All data were tested for significance using unpaired Student's t-test or ANOVA (one way \& two ways). Data were analyzed by Excel 2010 or GraphPad Prism Software, USA. A value of $P$ value $\leq 0.05$ was statistically significant. For further details please see Supplementary Tables, where the significance of time and group factor are displayed for all experiments.

Received: 9 November 2020; Accepted: 8 March 2021

Published online: 29 March 2021

\section{References}

1. Nobel, Y. R. et al. Metabolic and metagenomic outcomes from early-life pulsed antibiotic treatment. Nat. Commun. 6, 7486. https:// doi.org/10.1038/ncomms8486 (2015).

2. Lobionda, S., Sittipo, P., Kwon, H. Y. \& Lee, Y. K. The role of gut microbiota in intestinal inflammation with respect to diet and extrinsic stressors. Microorganisms 7, 271. https://doi.org/10.3390/microorganisms7080271 (2019).

3. Molodecky, N. A. et al. Increasing incidence and prevalence of the inflammatory bowel diseases with time, based on systematic review. Gastroenterology 142, 46-54. https://doi.org/10.1053/j.gastro.2011.10.001 (2012).

4. Rutter, M. D. et al. Thirty-year analysis of a colonoscopic surveillance program for neoplasia in ulcerative colitis. Gastroenterology 130, 1030-1038. https://doi.org/10.1053/j.gastro.2005.12.035 (2006).

5. Ullman, T. A. \& Itzkowitz, S. H. Intestinal inflammation and cancer. Gastroenterology 140, 1807-1816. https://doi.org/10.1053/j. gastro.2011.01.057 (2011).

6. Pahl, H. L. Activators and target genes of Rel/NF-kappaB transcription factors. Oncogene 18, 6853-6866. https://doi.org/10.1038/ sj.onc.1203239 (1999).

7. Jurjus, A. et al. Inflammatory bowel disease, colorectal cancer and type 2 diabetes mellitus: the links. BBA Clin. 5, 16-24. https:// doi.org/10.1016/j.bbacli.2015.11.002 (2015).

8. Morgan, D., Garg, M., Tergaonkar, V., Tan, S. Y. \& Sethi, G. Pharmacological significance of the non-canonical NF- $\kappa B$ pathway in tumorigenesis. Biochim. Biophys. Acta Rev. Cancer 1874, 188449. https://doi.org/10.1016/j.bbcan.2020.188449 (2020).

9. Ambriz-Pérez, D. L., Leyva-López, N., Gutierrez-Grijalva, E. P. \& Heredia, J. B. Phenolic compounds: natural alternative in inflammation treatment. A review. Cogent Food Agric. 2, 1131412. https://doi.org/10.1080/23311932.2015.1131412 (2016).

10. Sánchez-González, C., Izquierdo-Pulido, M. \& Noé, V. Urolithin A causes p21 up-regulation in prostate cancer cells. Eur. J. Nutr. 55, 1099-1112 (2016).

11. Koch, W. Dietary polyphenols-important non-nutrients in the prevention of chronic noncommunicable diseases. A systematic review. Nutrients 11, 1039. https://doi.org/10.3390/nu11051039 (2019).

12. Heber, D. Multitargeted therapy of cancer by ellagitannins. Cancer Lett. 269, 262-268. https://doi.org/10.1016/j.canlet.2008.03. 043 (2008)

13. Alasalvar, C. \& Bolling, B. W. Review of nut phytochemicals, fat-soluble bioactives, antioxidant components and health effects. Br. J. Nutr. 113(Suppl 2), S68-S78. https://doi.org/10.1017/S0007114514003729 (2015).

14. Seeram, N. P. et al. Pomegranate juice ellagitannin metabolites are present in human plasma and some persist in urine for up to 48 h. J. Nutr. 136, 2481 (2006).

15. Cerdá, B., Periago, P., Espín, J. C. \& Tomás-Barberán, F. A. Identification of urolithin a as a metabolite produced by human colon microflora from ellagic acid and related compounds. J. Agric. Food Chem. 53, 5571-5576. https://doi.org/10.1021/jf050384i (2005).

16. Selma, M. V., Beltrán, D., García-Villalba, R., Espín, J. C. \& Tomás-Barberán, F. A. Description of urolithin production capacity from ellagic acid of two human intestinal Gordonibacter species. Food Funct. 5, 1779-1784. https://doi.org/10.1039/c4fo00092g (2014).

17. Cerdá, B., Llorach, R., Cerón, J. J., Espín, J. C. \& Tomás-Barberán, F. A. Evaluation of the bioavailability and metabolism in the rat of punicalagin, an antioxidant polyphenol from pomegranate juice. Eur. J. Nutr. 42, 18-28. https://doi.org/10.1007/s00394-0030396-4 (2003). 
18. Espín, J. C., Larrosa, M., García-Conesa, M. T. \& Tomás-Barberán, F. Biological significance of urolithins, the gut microbial ellagic acid-derived metabolites: the evidence so far. Evid. Based Complement. Altern. Med. 1-15, 2013. https://doi.org/10.1155/2013/ 270418 (2013).

19. Garcia-Villalba, R. et al. Gastrointestinal simulation model TWIN-SHIME shows differences between human urolithinmetabotypes in gut microbiota composition, pomegranate polyphenol metabolism, and transport along the intestinal tract. $J$. Agric. Food Chem. 65, 5480-5493. https://doi.org/10.1021/acs.jafc.7b02049 (2017).

20. Smith, P. M. et al. The microbial metabolites, short-chain fatty acids, regulate colonic Treg cell homeostasis. Science 341, 569-573. https://doi.org/10.1126/science.1241165 (2013).

21. Atarashi, K. et al. Induction of colonic regulatory T cells by indigenous Clostridium species. Science 331, 337-341. https://doi.org/ 10.1126/science.1198469 (2011).

22. Tanca, A. et al. Clostridial butyrate biosynthesis enzymes are significantly depleted in the gut microbiota of nonobese diabetic mice. $m$ Sphere https://doi.org/10.1128/mSphere.00492-18 (2018).

23. Mariño, E. et al. Gut microbial metabolites limit the frequency of autoimmune T cells and protect against type 1 diabetes. Nat. Immunol. 18, 552-562. https://doi.org/10.1038/ni.3713 (2017).

24. Sharif, O., Bolshakov, V. N., Raines, S., Newham, P. \& Perkins, N. D. Transcriptional profiling of the LPS induced NF- $\kappa$ B response in macrophages. BMC Immunol. 8, 1 (2007).

25. Fujiwara, N. \& Kobayashi, K. Macrophages in inflammation. Curr. Drug Targets Inflamm. Allergy 4, 281-286. https://doi.org/10. 2174/1568010054022024 (2005)

26. Takashiba, S. et al. Differentiation of monocytes to macrophages primes cells for lipopolysaccharide stimulation via accumulation of cytoplasmic nuclear factor kappaB. Infect. Immun. 67, 5573-5578 (1999).

27. McCarthy, C. G. et al. Toll-like receptors and damage-associated molecular patterns: novel links between inflammation and hypertension. Am. J. Physiol. Heart Circ. Physiol. 306, H184-196. https://doi.org/10.1152/ajpheart.00328.2013 (2014).

28. Akira, S., Takeda, K. \& Kaisho, T. Toll-like receptors: critical proteins linking innate and acquired immunity. Nat. Immunol. 2, 675-680. https://doi.org/10.1038/90609 (2001).

29. Takeuchi, O. \& Akira, S. Pattern recognition receptors and inflammation. Cell 140, 805-820. https://doi.org/10.1016/j.cell.2010. $01.022(2010)$

30. Verstak, B. et al. MyD88 adapter-like (Mal)/TIRAP interaction with TRAF6 is critical for TLR2- and TLR4-mediated NF-kappaB proinflammatory responses. J. Biol. Chem. 284, 24192-24203. https://doi.org/10.1074/jbc.M109.023044 (2009).

31. Mukherjee, S., Karmakar, S. \& Babu, S. P. S. TLR2 and TLR4 mediated host immune responses in major infectious diseases: a review. Braz. J. Infect. Dis. 20, 193-204. https://doi.org/10.1016/j.bjid.2015.10.011 (2016).

32. Ishmael, F. T. et al. (2008) Role of the RNA-binding protein tristetraprolin in glucocorticoid-mediated gene regulation. J. Immunol. (Baltimore) 180, 8342-8353. https://doi.org/10.4049/jimmunol.180.12.8342 (2008).

33. Fan, J. et al. Chemokine transcripts as targets of the RNA-binding protein HuR in human airway epithelium. J. Immunol. 186, 2482. https://doi.org/10.4049/jimmunol.0903634 (2011).

34. Kirtonia, A., Sethi, G. \& Garg, M. The multifaceted role of reactive oxygen species in tumorigenesis. Cell. Mol. Life Sci. 77, 4459-4483. https://doi.org/10.1007/s00018-020-03536-5 (2020).

35. Gordeeva, A. V., Zvyagilskaya, R. A. \& Labas, Y. A. Cross-talk between reactive oxygen species and calcium in living cells. Biochem. Biokhimiia 68, 1077-1080. https://doi.org/10.1023/a:1026398310003 (2003).

36. Redon, C. E. et al. Tumors induce complex DNA damage in distant proliferative tissues in vivo. Proc. Natl. Acad. Sci. 107, 1799217997 (2010).

37. Imlay, J. A., Chin, S. M. \& Linn, S. Toxic DNA damage by hydrogen peroxide through the Fenton reaction in vivo and in vitro. Science 240, 640-642 (1988)

38. Mantovani, A. et al. The chemokine system in diverse forms of macrophage activation and polarization. Trends Immunol. 25, 677-686. https://doi.org/10.1016/j.it.2004.09.015 (2004).

39. Fukao, T. \& Koyasu, S. PI3K and negative regulation of TLR signaling. Trends Immunol. 24, 358-363. https://doi.org/10.1016/ s1471-4906(03)00139-x (2003).

40. Song, G., Ouyang, G. \& Bao, S. The activation of Akt/PKB signaling pathway and cell survival. J. Cell. Mol. Med. 9, 59-71. https:// doi.org/10.1111/j.1582-4934.2005.tb00337.x (2005).

41. Mogensen, T. H. Pathogen recognition and inflammatory signaling in innate immune defenses. Clin. Microbiol. Rev. 22, 240. https://doi.org/10.1128/CMR.00046-08 (2009).

42. Lester, S. N. \& Li, K. Toll-like receptors in antiviral innate immunity. J. Mol. Biol. 426, 1246-1264. https://doi.org/10.1016/j.jmb. 2013.11.024 (2014).

43. McCoy, C. E. \& O’neill, L. The role of toll-like receptors in macrophages. Front. Biosci. 13, 62-70 (2008).

44. Bagaev, A. V. et al. Elevated pre-activation basal level of nuclear NF- $\mathrm{kB}$ in native macrophages accelerates LPS-induced translocation of cytosolic NF- $\kappa B$ into the cell nucleus. Sci. Rep. 9, 4563. https://doi.org/10.1038/s41598-018-36052-5 (2019).

45. Soares, J.-B., Pimentel-Nunes, P., Roncon-Albuquerque, R. \& Leite-Moreira, A. The role of lipopolysaccharide/toll-like receptor 4 signaling in chronic liver diseases. Hepatol. Int. 4, 659-672. https://doi.org/10.1007/s12072-010-9219-x (2010).

46. Szajnik, M. et al. TLR4 signaling induced by lipopolysaccharide or paclitaxel regulates tumor survival and chemoresistance in ovarian cancer. Oncogene 28, 4353-4363. https://doi.org/10.1038/onc.2009.289 (2009).

47. Vergadi, E., Ieronymaki, E., Lyroni, K., Vaporidi, K. \& Tsatsanis, C. Akt signaling pathway in macrophage activation and M1/M2 polarization. J. Immunol. (Baltimore) 198, 1006-1014. https://doi.org/10.4049/jimmunol.1601515 (2017).

48. Momen-Heravi, F. \& Bala, S. miRNA regulation of innate immunity. J. Leukoc. Biol. 103, 1205-1217. https://doi.org/10.1002/jlb. 3 mir1117-459r (2018).

49. Larrosa, M. et al. Anti-inflammatory properties of a pomegranate extract and its metabolite urolithin-A in a colitis rat model and the effect of colon inflammation on phenolic metabolism. J. Nutr. Biochem. 21, 717-725. https://doi.org/10.1016/j.jnutbio.2009. $04.012(2010)$.

50. Arpaia, N. et al. Metabolites produced by commensal bacteria promote peripheral regulatory T-cell generation. Nature 504, 451-455. https://doi.org/10.1038/nature12726 (2013).

51. Tomas-Barberan, F. A., Garcia-Villalba, R., Gonzalez-Sarrias, A., Selma, M. V. \& Espin, J. C. Ellagic acid metabolism by human gut microbiota: consistent observation of three urolithin phenotypes in intervention trials, independent of food source, age, and health status. J. Agric. Food Chem. 62, 6535-6538. https://doi.org/10.1021/jf5024615 (2014).

52. Liu, F. et al. Inhibition of polypeptide N-acetyl- $\alpha$-galactosaminyltransferases is an underlying mechanism of dietary polyphenols preventing colorectal tumorigenesis. Bioorg. Med. Chem. 27, 3372-3382. https://doi.org/10.1016/j.bmc.2019.06.020 (2019).

53. Mohammed Saleem, Y. I., Albassam, H. \& Selim, M. Urolithin A induces prostate cancer cell death in p53-dependent and in p53-independent manner. Eur. J. Nutr. https://doi.org/10.1007/s00394-019-02016-2 (2019).

54. Singh, R. et al. Enhancement of the gut barrier integrity by a microbial metabolite through the Nrf2 pathway. Nat. Commun. 10, 89. https://doi.org/10.1038/s41467-018-07859-7 (2019).

55. Calin, G. A. et al. MicroRNA profiling reveals distinct signatures in B cell chronic lymphocytic leukemias. Proc. Natl. Acad. Sci. 101, 11755-11760 (2004).

56. Davidson-Moncada, J., Papavasiliou, F. N. \& Tam, W. MicroRNAs of the immune system: roles in inflammation and cancer. Ann. N.Y. Acad. Sci. 1183, 183-194. https://doi.org/10.1111/j.1749-6632.2009.05121.x (2010). 
57. Chaudhuri, A. A. et al. MicroRNA-125b potentiates macrophage activation. J. Immunol. (Baltimore) 187, 5062-5068. https://doi. org/10.4049/jimmunol.1102001 (2011).

58. Bazzoni, F. et al. Induction and regulatory function of miR-9 in human monocytes and neutrophils exposed to proinflammatory signals. Proc. Natl. Acad. Sci. 106, 5282-5287 (2009).

59. Rahman, W. \& Dickenson, A. H. Voltage gated sodium and calcium channel blockers for the treatment of chronic inflammatory pain. Neurosci. Lett. 557, 19-26. https://doi.org/10.1016/j.neulet.2013.08.004 (2013).

60. Clapham, D. E. Calcium signaling. Cell 131, 1047-1058 (2007).

61. Liu, T. et al. Role of reactive oxygen species-mediated MAPK and NF-kB activation in polygonatum cyrtonema lectin-induced apoptosis and autophagy in human lung adenocarcinoma A549 cells. J. Biochem. 160, 315-324. https://doi.org/10.1093/jb/mvw040 (2016).

62. Liu, C. M., Sun, Y. Z., Sun, J. M., Ma, J. Q. \& Cheng, C. Protective role of quercetin against lead-induced inflammatory response in rat kidney through the ROS-mediated MAPKs and NF-kB pathway. Biochim. Biophys. Acta 1693-1703, 2012. https://doi.org/ 10.1016/j.bbagen.2012.06.011 (1820).

63. Chumduri, C., Gurumurthy, R. K., Zadora, P. K., Mi, Y. \& Meyer, T. F. Chlamydia infection promotes host DNA damage and proliferation but impairs the DNA damage response. Cell. Host Microbe. 13, 746-758. https://doi.org/10.1016/j.chom.2013.05. 010 (2013).

64. Qiao, W. et al. Lipopolysaccharide-induced DNA damage response activates nuclear factor $\mathrm{kB}$ signalling pathway via GATA4 in dental pulp cells. Int. Endod. J. 52, 1704-1715. https://doi.org/10.1111/iej.13180 (2019).

65. Pelzl, L. et al. Lithium sensitive ORAI1 expression, store operated Ca2+ entry and suicidal death of neurons in chorea-acanthocytosis. Sci. Rep. 7, 6457. https://doi.org/10.1038/s41598-017-06451-1 (2017).

66. Zhang, S. et al. Epigallocatechin-3-gallate (EGCG) up-regulates miR-15b expression thus attenuating store operated calcium entry (SOCE) into murine CD4 + T cells and human leukaemic T cell lymphoblasts. Oncotarget 8, 89500-89514 (2017).

67. Singh, Y. et al. Enhanced reactive oxygen species production, acidic cytosolic $\mathrm{pH}$ and upregulated $\mathrm{Na}+\mathrm{H}+$ exchanger (NHE) in dicer deficient CD4+ T cells. Cell. Physiol. Biochem. Int. J. Exp. Cell. Physiol. Biochem. Pharmacol. 42, 1377-1389. https://doi.org/ $10.1159 / 000479201(2017)$.

68. Zhang, S., Zeng, Y., Yi, X. \& Zhang, Y. Selection of suitable reference genes for quantitative RT-PCR normalization in the halophyte Halostachys caspica under salt and drought stress. Sci. Rep. 6, 30363-30363. https://doi.org/10.1038/srep30363 (2016).

69. Singh, Y. et al. Alkaline cytosolic $\mathrm{pH}$ and high sodium hydrogen exchanger 1 (NHE1) activity in Th9 Cells. J. Biol. Chem. 291, 23662-23671. https://doi.org/10.1074/jbc.M116.730259 (2016).

\section{Acknowledgements}

We are thankful to the Arab Republic of Egypt (Egyptian Culture Affairs \& Missions Sector) Scholarship to K.N.M.A. We acknowledge support by Open Access Publishing Fund of University of Tübingen.

\section{Author contributions}

S.B.H and K.N.M.A designed the study; F.L. and S.B.H. provided suggestions and lab facility. K.N.M.A conducted the experiments, analysed the results and wrote the manuscript, M.Z.K helped in the immunofluorescence images. F.L and S.B.H. reviewed the manuscript.

\section{Funding}

Open Access funding enabled and organized by Projekt DEAL.

\section{Competing interests}

The authors declare no competing interests.

\section{Additional information}

Supplementary Information The online version contains supplementary material available at https://doi.org/ 10.1038/s41598-021-86514-6.

Correspondence and requests for materials should be addressed to S.B.-H.

Reprints and permissions information is available at www.nature.com/reprints.

Publisher's note Springer Nature remains neutral with regard to jurisdictional claims in published maps and institutional affiliations.

Open Access This article is licensed under a Creative Commons Attribution 4.0 International

License, which permits use, sharing, adaptation, distribution and reproduction in any medium or format, as long as you give appropriate credit to the original author(s) and the source, provide a link to the Creative Commons licence, and indicate if changes were made. The images or other third party material in this article are included in the article's Creative Commons licence, unless indicated otherwise in a credit line to the material. If material is not included in the article's Creative Commons licence and your intended use is not permitted by statutory regulation or exceeds the permitted use, you will need to obtain permission directly from the copyright holder. To view a copy of this licence, visit http://creativecommons.org/licenses/by/4.0/.

(c) The Author(s) 2021 